\title{
Pflegerische Versorgung in der ersten Welle der COVID-19-Pandemie
}

Miriam Räker, Jürgen Klauber und Antje Schwinger

\section{Inhaltsverzeichnis}

\subsection{Einleitung - 35}

3.2 Methodik und Deskription der Stichprobe - 36

3.2.1 Methodik - 36

3.2.2 Deskription und Repräsentativität der Stichprobe - 37

3.3 Veränderung der pflegerischen Versorgung und der Kontaktmöglichkeiten im häuslichen Setting - 41

3.3.1 Inanspruchnahme und Veränderung von Unterstützungsleistungen - 41

3.3.2 Veränderung der Kontaktmöglichkeiten - 42

3.3.3 Bewertung der pflegerischen Versorgung - 44

3.3.4 Belastungssituation der Pflegeperson - 45

3.3.5 Zusammenfassung und Zwischenfazit zur pflegerischen Versorgung im häuslichen Setting - 46

3.4 Veränderung der pflegerischen Versorgung und der Kontaktmöglichkeiten im vollstationären Setting - 47

3.4.1 Veränderung der Kontaktmöglichkeiten - 47

3.4.2 Bewertung der pflegerischen Versorgung - 49

3.4.3 Belastungssituation der Bezugsperson - 50 
3.4.4 Fokus ärztliche Versorgung in Pflegeeinrichtungen - 52

3.4.5 Zusammenfassung und Zwischenfazit zur pflegerischen Versorgung im vollstationären Setting - 55

3.5 Zusammenfassung und Fazit - 56

Literatur - 57 


\section{- Zusammenfassung}

Im Rahmen der COVID-19-Pandemie gelten pflegebedürftige Menschen als besonders vulnerabel und anfällig für schwere Krankheitsverläufe. In der Folge zielten und zielen viele Maßnahmen zur Eindämmung der Pandemie auf den Schutz dieser Bevölkerungsgruppe, in erster Linie einhergehend mit Kontaktbeschränkungen. Gleichzeitig ist anzunehmen, dass die Pandemiebedingungen und die ergriffenen Maßnahmen Einfluss auf die pflegerische und gesundheitliche Versorgungssituation der pflegebedürftigen Personen haben. Mittels einer Online-Befragung wurden Pflegeund Bezugspersonen aus dem ambulanten und dem vollstationären Pflegesetting retrospektiv zu ihrer Wahrnehmung der Lebens- und Versorgungsbedingungen von ihnen nahestehenden pflegebedürftigen Menschen während der ersten Welle der COVID-19-Pandemie befragt. Die Analysen zeigen auf, dass es neben starken Einschränkungen der sozialen Teilhabe der pflegebedürftigen Personen auch zu Veränderungen in den Pflegearrangements gekommen ist. Die Befragten spiegeln deutliche soziale und psychische Belastungen der pflegebedürftigen Menschen wie auch ihrer gesundheitlichen bzw. ärztlichen Versorgung wider. Auch die Betreuenden selber zeigen deutlich gestiegene emotionale Belastungen.

In the context of the COVID 19 pandemic, people in need of care are considered particularly vulnerable and susceptible to severe courses of the disease. As a result, many pandemic containment measures were and are aimed at protecting this group, primarily accompanied by contact restrictions. At the same time, the pandemic conditions and the measures taken probably have an influence on the care and health care situation of the persons in need of care. By means of an online survey, caregivers from outpatient and inpatient care settings were retrospectively asked about their perception of the living and care conditions of people in need of care close to them during the first wave of the COVID 19 pandemic. The analyses show that, in addition to severe restrictions in the social participation of the persons in need of care, care arrangements have also changed. The respondents reflect clear social and psychological burdens on the people in need of care, their health and their medical care. The carers themselves also experience a clear increase in emotional stress.

\subsection{Einleitung}

Ältere sowie pflegebedürftige Menschen respektive Menschen mit Grunderkrankungen werden mit einem schweren COVID-19-Verlauf in Verbindung gebracht (Gardner et al. 2020). Aufgrund des Mangels an wirksamen Medikamenten und der zunächst fehlenden Verfügbarkeit von Schutzimpfungen standen nicht nur während der ersten Pandemiewelle in Deutschland im Frühjahr 2020, sondern auch im weiteren Verlauf der Pandemie primär Public-Health-Interventionen zum Schutz der Allgemeinbevölkerung sowie im besonderen Maße der vulnerablen Bevölkerungsgruppen im Vordergrund. Diese umfassen physisches Abstandhalten in Form der Reduzierung bzw. Vermeidung von persönlichen Kontakten, Hygienemaßnahmen, gezieltes Testen sowie Isolations- und Quarantänemaßnahmen (Müller et al. 2020). Die damit einhergehenden Maßnahmen belasten und belasteten das private, öffentliche und wirtschaftliche Leben und stellen somit eine besondere Herausforderung für eine bedarfsgerechte Versorgung und insbesondere auch den Schutz von pflegebedürftigen Menschen dar (Kessler et al. 2020b). Auf die potenziellen körperlichen, sozialen, kognitiven und emotionalen sowie versorgungsbezogenen Gefahren u. a. der sozialen Isolation sowie anderer Schutzmaßnahmen für Menschen allgemein sowie vor allem für ältere und pflegebedürftige Menschen wurde bereits vielfach verwiesen (Kessler et al. 2020a; Röhr et al. 2020). Des Weiteren ist davon auszugehen, dass die Herausforderungen in der häuslichen 
Pflege und die Belastungen der Pflegepersonen, die seit Jahren hoch sind, durch die COVID-19-Pandemie verschärft wurden bzw. werden (Eggert et al. 2020; Horn und Schweppe 2020; Rothgang et al. 2020). Ähnliche Annahmen gelten für die stationäre Langzeitpflege und wurden für diese - primär für das Pflegepersonal - bereits bestätigt (Eggert und Teubner 2021).

Die vorliegende Befragung fokussiert die Folgen der mit der COVID-19-Pademie einhergehenden Veränderungen für ambulant und vollstationär Pflegebedürftige in der ersten Pandemiewelle von März bis Juni 2020. Mittels einer onlinegestützten Proxy-Befragung (Stellvertreter-Befragung) soll die Lebensund Versorgungssituation von pflegebedürftigen Menschen während des ersten Lockdowns erfasst werden. Zum einen wurden Fragen gestellt, die die Wahrnehmung der Pflegeund Bezugspersonen pflegebedürftiger Menschen hinsichtlich der Pandemie und der damit einhergehenden Maßnahmen erfassen sollen. Zum anderen liegt der Schwerpunkt der Untersuchung auf der pflegerischen und gesundheitlichen respektive ärztlichen Versorgungsund Lebenssituation der pflegebedürftigen Personen während der ersten Pandemie-Welle. Neben dem emotionalen und gesundheitlichen Zustand der pflegebedürftigen Personen wurden auch die Belastungen der Pflege- bzw. Bezugspersonen erfasst.

\subsection{Methodik und Deskription der Stichprobe}

\subsubsection{Methodik}

Mithilfe des forsa.omninet-Panels wurde eine Online-Befragung von Pflegepersonen (häusliches Versorgungssetting) und Bezugspersonen (vollstationäres Versorgungssetting) durchgeführt. Das forsa.omninet-Panel umfasst ca. 75.000 Teilnehmende, die Panelisten wurden über bevölkerungsrepräsentative Telefoninterviews auf Basis von Zufallsstich- proben rekrutiert. Für das ambulante Setting wurden Personen in Deutschland ab 18 Jahre eingeschlossen, die - laut Selbstauskunft „Pflegeperson“ einer pflegebedürftigen Person mit anerkanntem Pflegegrad der Pflegeversicherung sind, die nicht im Pflegeheim wohnt. Die zu pflegende Person musste nicht im selben Haushalt wie die befragte Person leben. Für das stationäre Setting wurden Personen in Deutschland ab 18 Jahre eingeschlossen, die - laut Selbstauskunft - „Bezugsperson“ mit regelmäßigem Kontakt zu einer pflegebedürftigen und stationär gepflegten Person sind. Feldzugang und Methodik entsprechen einer Erhebung des Wissenschaftlichen Instituts der AOK (WIdO) aus dem Jahr 2019 zur Frage finanzieller und zeitlicher Eigenleistungen von Pflegehaushalten (Räker et al. 2020). Diese wird als Referenzstudie für das ambulante Setting herangezogen.

Insgesamt enthielt die Nettostichprobe 1.012 Befragte. Für das ambulante Setting konnte eine Fallzahl von 505 Fällen und für das stationäre Setting eine Fallzahl von 507 Fällen realisiert werden. Die Erhebung erfolgte im Zeitraum vom 26. Oktober bis 13. November 2020. Sie fand damit zu Beginn der zweiten Pandemiewelle und vor dem Hintergrund eines Teil-Lockdowns ab dem 2. November statt (Bundesregierung 2020). Gegenstand der Befragung war jedoch ausschließlich die erste Welle der COVID-19-Pandemie, die in den Fragestellungen auf den Zeitraum März bis Mai 2020 begrenzt wurde.

Zur Sicherung der Repräsentativität der Ergebnisse wurde eine retrospektive StrukturGewichtung der Stichprobenergebnisse nach Pflegegrad, Geschlecht und Alter der gepflegten Person auf Grundlage der zu dem Zeitpunkt aktuellen Pflegestatistik vorgenommen (Statistisches Bundesamt 2018). Zur Einordnung der Repräsentativität der Stichprobe für die ambulante Versorgung wurden vorrangig die Studien von Kantar (2019) sowie Räker et al. (2020) herangezogen. Mit Blick auf die Einordnung der Erkenntnisse im Kontext der COVID-19-Pandemie werden für das ambulante Setting drei weitere Befragungen heran- 
gezogen (Eggert et al. 2020; Rothgang et al. 2020; Horn und Schweppe 2020). Allerdings fokussiert keine dieser sowie weitere verfügbare Untersuchungen explizit die gesundheitliche Lage der Pflegebedürftigen. Verfügbare Studien aus der vollstationären Langzeitversorgung nehmen primär das Pflegepersonal, nicht jedoch die Pflegebedürftigen oder ihre Bezugspersonen in den Blick, wie z. B. Eggert und Teubner (2021).

\subsubsection{Deskription und Repräsentativität der Stichprobe}

Aufgrund der vorgenommenen Gewichtung entspricht die Alters-, Geschlechts-, Pflegegrad- und Versorgungsartverteilung der - indirekt über die Pflege- bzw. Bezugsperson betrachteten Pflegebedürftigen derjenigen der bundesweit ambulant und stationär Pflegebedürftigen (• Tab. 3.1).
Der Anteil dementiell Erkrankter im häuslichen Setting liegt mit $20 \%$ ähnlich hoch wie in anderen Erhebungen. Der Blick auf die Stichprobe des vollstationären Settings zeigt, dass hier erwartungsgemäß der Anteil mit einer dementiellen Erkrankung mit 50\% deutlich höher liegt (•Tab. 3.1). Hervorzuheben ist, dass nur $44 \%$ der Befragten im ambulanten Setting im selben Haushalt wie die pflegebedürftige Person leben, was im Vergleich zu den Referenzstudien ein geringerer Anteil ist (Kantar 2019: 65 \%; Räker et al. 2020: 50,3\%; Rothgang et al. 2020: $81 \%$ ) (• Tab. 3.1).

$\mathrm{Da}$ es für die Bewertung der Aussagen zur Situation der Pflegebedürftigen in der Pandemie relevant ist, wird an dieser Stelle auf das Verwandtschaftsverhältnis bzw. die Beziehung und Kontaktintensität der befragten Personen zur pflegebedürftigen Person eingegangen. Hier unterscheiden sich das ambulante und das stationäre Setting deutlich: Lediglich eine Minderheit der befragten Bezugspersonen von vollstationär Pflegebedürftigen $(3,2 \%)$ betreut ihren eigenen Lebenspartner, während

- Tab. 3.1 Charakterisierung der Stichprobe

\begin{tabular}{|c|c|c|c|}
\hline \multicolumn{2}{|l|}{ Pflegebedürftige Person } & Ambulant & Stationär \\
\hline \multirow{5}{*}{$\begin{array}{l}\text { Alter } \\
\mathrm{N} \text { ambulant }=502 ; \mathrm{N} \text { stationär }=505\end{array}$} & 0-17 Jahre & 7,8 & 0,1 \\
\hline & 18-59 Jahre & 10,3 & 4,3 \\
\hline & 60-79 Jahre & 16,8 & 12,7 \\
\hline & $80+$ & 65,1 & 82,8 \\
\hline & Mittelwert & 71,5 & 82,1 \\
\hline \multirow{2}{*}{$\begin{array}{l}\text { Geschlecht } \\
\mathrm{N} \text { ambulant }=504 ; \mathrm{N} \text { stationär }=505\end{array}$} & Männlich & 39,5 & 29,4 \\
\hline & Weiblich & 60,5 & 70,6 \\
\hline \multirow{5}{*}{$\begin{array}{l}\text { Pflegegrad } \\
\mathrm{N} \text { ambulant }=500 ; \mathrm{N} \text { stationär }=478\end{array}$} & Pflegegrad 1 & 1,5 & 0,8 \\
\hline & Pflegegrad 2 & 53,6 & 20,7 \\
\hline & Pflegegrad 3 & 29,5 & 31,7 \\
\hline & Pflegegrad 4 & 11,9 & 30,1 \\
\hline & Pflegegrad 5 & 3,5 & 16,7 \\
\hline
\end{tabular}


Tab. 3.1 (Fortsetzung)

\begin{tabular}{|c|c|c|c|}
\hline \multicolumn{2}{|l|}{ Pflegebedürftige Person } & Ambulant & Stationär \\
\hline \multirow{2}{*}{$\begin{array}{l}\text { Demenz } \\
\mathrm{N} \text { ambulant }=498 ; \mathrm{N} \text { stationär }=501\end{array}$} & $\mathrm{Ja}$ & 19,6 & 49,6 \\
\hline & Nein & 80,4 & 50,4 \\
\hline
\end{tabular}

\section{Pflegebedürftige Person (stationär)}

Bisherige Dauer des Aufenthalts in der Pflegeinrichtung $\mathrm{N}=503$

Größe der Pflegeeinrichtung $\mathrm{N}=255$

\section{Lebt die pflegebedürftige Person} in einem Einzelzimmer $\mathrm{N}=505$

Entfernung zwischen Pflegeeinrichtung und Wohnort der Bezugsperson $\mathrm{N}=502$
Höchstens ein Jahr

1 Jahr weniger 2 Jahre

2 Jahre weniger 3 Jahre

3 Jahre weniger 4 Jahre

4 Jahren weniger 5 Jahre

5 und mehr Jahre

50 oder weniger Plätze/Bew.

50 bis 100 Plätze/Bew.

mehr als 100 Plätze/Bew.

$\mathrm{Ja}$

Nein

Bis zu $30 \mathrm{~min}$

Bis zu $60 \mathrm{~min}$

Bis zu 90 min

Mehr als $90 \mathrm{~min}$

$$
6,0
$$

20,2

23,4

52,9

23,8

83,3

16,7

63,6

19,9

11,2

\section{Pflegebedürftige Person und Pflegeperson (ambulant)}

Pflegeperson und pflegebedürftige

43,6

Person leben im selben $\mathrm{HH} \mathrm{N}=505$

Pflege-Report 2021

dies bei den ambulant Pflegenden jeden fünften Fall betrifft $(21,9 \%)$ (- Abb. 3.1). Die befragten Bezugspersonen, die Auskunft über die vollstationär Pflegebedürftigen geben, sind zu knapp einem Fünftel Freunde oder Nachbarn.

Um die Intensität der pflegerischen bzw. betreuenden Beziehung zu erfassen, wurde der typische wöchentliche Pflegeaufwand vor der COVID-19-Pandemie erfragt (• Abb. 3.2). Mehr als die Hälfte $(57,2 \%)$ der Pflegepersonen im ambulanten Setting kümmerte sich täg- lich um die pflegebedürftige Person, lediglich rund jede zehnte befragte Person $(9,5 \%)$ nur einmal pro Woche. Für das stationäre Setting gaben nur $8 \%$ der befragten Bezugspersonen an, sich täglich um die pflegebedürftige Person gekümmert zu haben, jede fünfte befragte Person $(21,7 \%)$ sogar weniger als einmal pro Woche (• Abb. 3.3).

Der Blick auf den formalen Bildungsabschluss zeigt, dass der Anteil der Pflegepersonen und Bezugspersonen mit einem hohen formalen Bildungsabschluss in der hiesigen Be- 


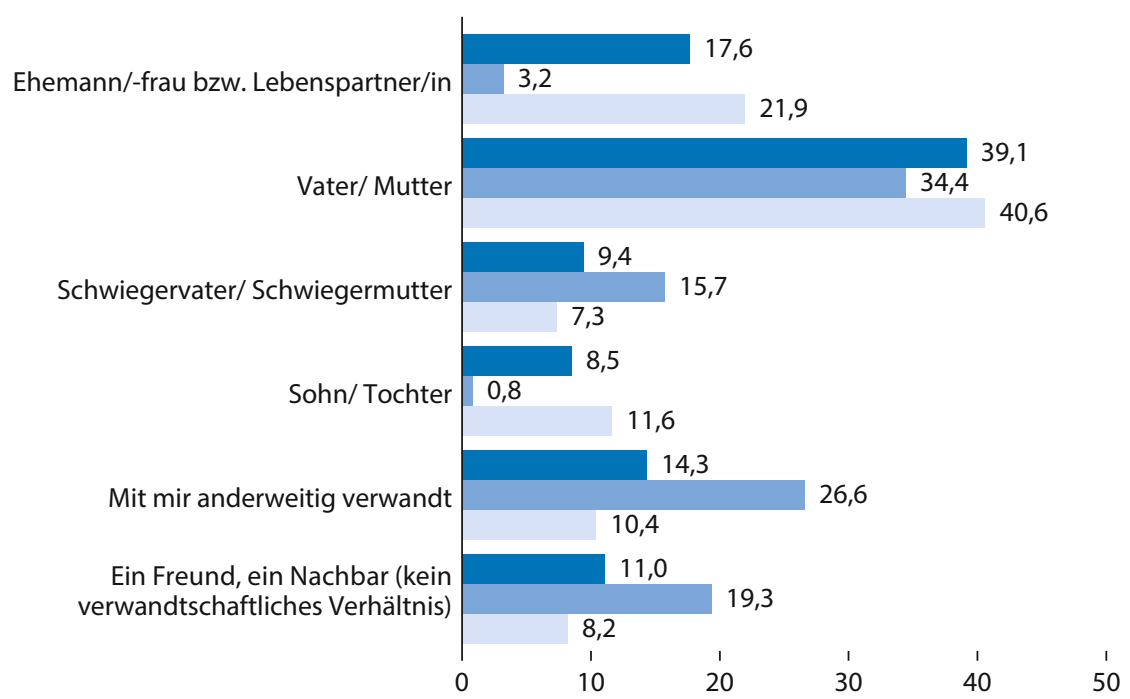

Insgesamt $\mathrm{N}=1007$ Bezugsperson (stationär) $\mathrm{N}=505 \quad$ Hauptpflegeperson (ambulant) $\mathrm{N}=499$

Pflege-Report 2021

- Abb. 3.1 Verwandtschaftliches oder sonstiges Verhältnis der befragten Person zur pflegebedürftigen Person, in \%

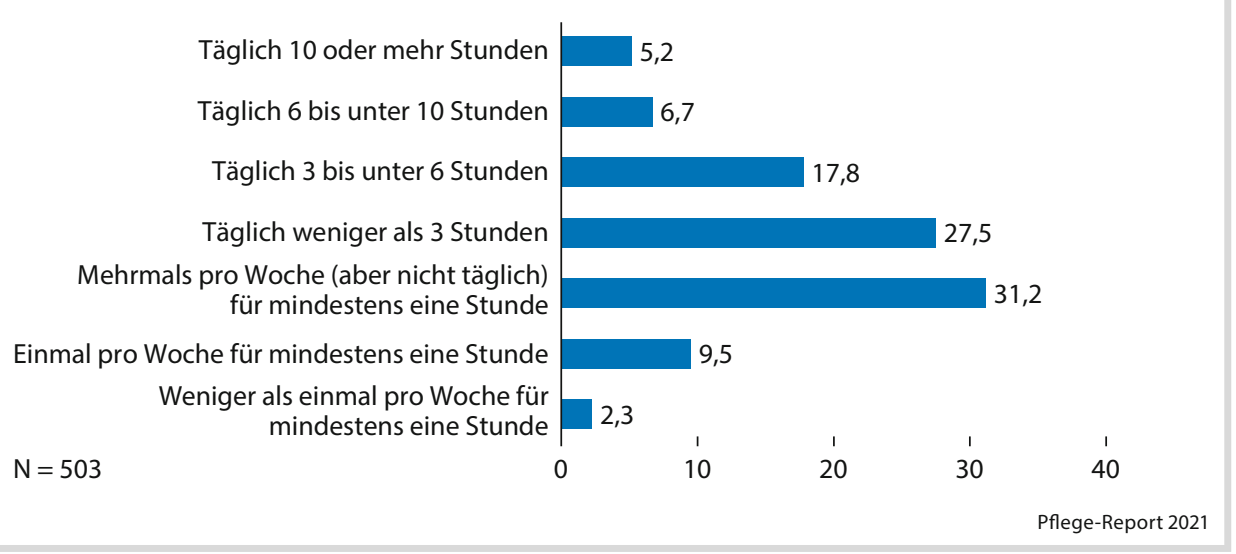

- Abb. 3.2 Häusliches Setting (in \%): Ungefähr wie viel Zeit in einer typischen Woche wendeten Sie vor Beginn der COVID-19-Pandemie dafür auf, sich um die pflegebedürftige Person zu kümmern?

fragung deutlich überrepräsentiert ist: $45 \%$ gaben als höchsten formalen Bildungsabschluss das Abitur (die allgemeine Hochschulreife) an (- Abb. 3.4). Bei Betrachtung des Anteils der Normalbevölkerung, der sich nicht in schulischer Ausbildung befindet, zeigt sich, dass nur rund $33 \%$ eine (Fach-)Hochschulreife er- langt haben (Statistisches Bundesamt 2019; eigene Berechnungen). Höher gebildete Personen sind in wissenschaftlichen Umfragen allgemein überrepräsentiert, insbesondere bei Online-Erhebungen (Schmidt 2018).

- Tab. 3.2 zeigt auf, dass auch das Haushaltseinkommen der Befragten überdurch- 


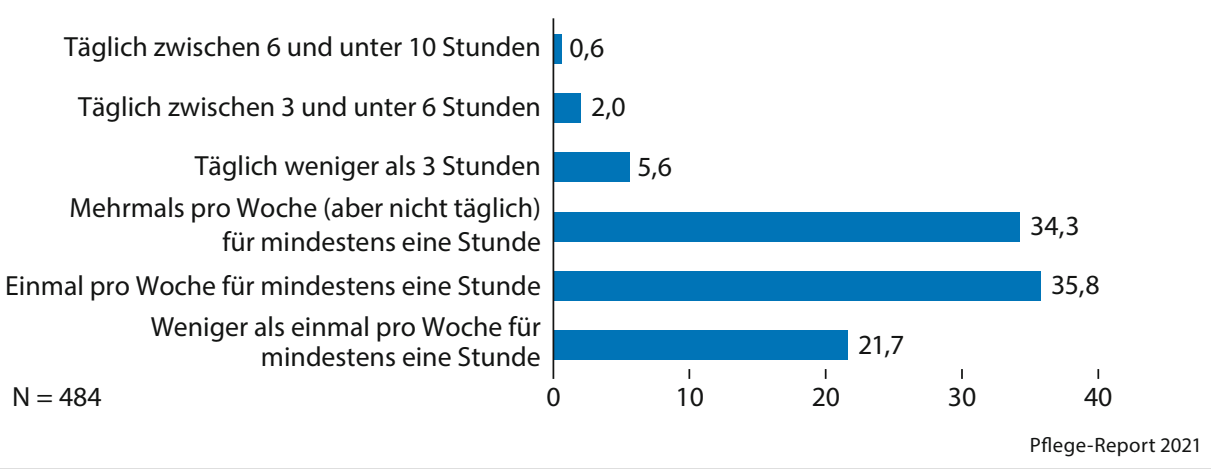

- Abb. 3.3 Vollstationäres Setting (in \%): Ungefähr wie viel Zeit in einer typischen Woche wendeten Sie vor Beginn der COVID-19-Pandemie dafür auf, sich um die pflegebedürftige Person zu kümmern?

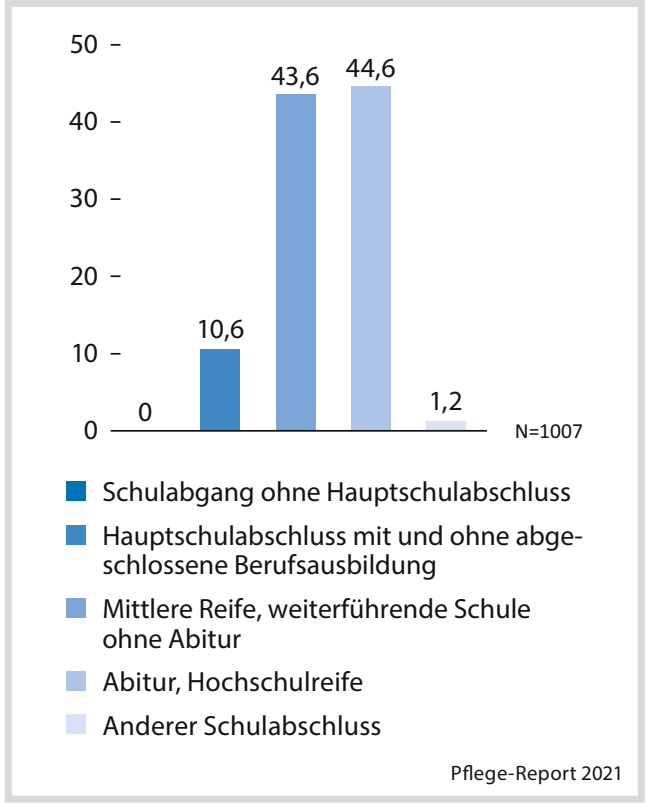

- Abb. 3.4 Übersicht zum Bildungsstand der befragten Person, in $\%$

schnittlich ist $-42 \%$ geben ein monatliches Nettoeinkommen von $4.000 €$ und mehr an. Dieses liegt nicht nur deutlich über dem Bundesdurchschnitt $(3.580 €$, durchschnittliches monatliches Haushaltsnettoeinkommen im Jahr 2019 (Statistisches Bundesamt 2020)), sondern auch über denen der Referenzbefra-
- Tab. 3.2 Haushaltsnettoeinkommen der befragten Personen insgesamt (Pflegeperson ambulant und Bezugsperson vollstationär, in \%)

\begin{tabular}{|l|l|}
\hline $\begin{array}{l}\text { Haushaltsnettoeinkommen } \\
\text { pro Monat }\end{array}$ & Anteil in \% \\
\hline Unter $1.000 €$ & 2,7 \\
\hline 1.000 bis unter $2.000 €$ & 13,8 \\
\hline 2.000 bis unter $4.000 €$ & 41,5 \\
\hline $4.000 €$ und mehr & 42,0 \\
\hline N $=907$ & \\
\hline Pflege-Report 2021 & \\
\hline
\end{tabular}

gungen für den ambulanten Kontext (Kantar 2019: $\leq 4.000=18 \%$; Horn und Schweppe 2020: $\leq 4.000=8,8 \%$ ).

In der Gesamtschau sind die für die Studie befragten Pflegepersonen für die ambulant Pflegebedürftigen bezogen auf Alter, Geschlecht und Pflegeschwere repräsentativ. Gleichwohl zeigen sich im Vergleich zu anderen Erhebungen Spezifika, die bei weiteren Interpretationen der Ergebnisse zu berücksichtigen sind. So ist das Bildungsniveau höher, sicherlich bedingt durch die gewählte Erhebungsmethode (- Abb. 3.4). Unterproportional vertreten sind auch gemeinsame Haushal- 
te von Pflegebedürftigen und Pflegepersonen (- Tab. 3.1). Für die im Folgenden berichteten Fremdeinschätzungen der Befragten über z. B. die seelische und körperliche Gesundheit der Pflegebedürftigen ist für das stationäre Versorgungssetting zudem zu beachten, dass jeder fünfte Befragte weniger als einmal pro Woche für eine Stunde Kontakt zum Pflegebedürftigen hatte (• Abb. 3.3). Zum Zeitpunkt der Befragung war nur ein kleiner Anteil der Befragten sowie der Pflegebedürftigen nachweislich mit dem Virus infiziert respektive infiziert gewesen - im vollstationären Setting immerhin 4 von 100 Pflegebedürftigen, im häuslichen Setting weniger als 1 von 100 . Die Befragten zeigen ferner - auch dies ist wichtig zur Einordnung der berichteten Einschätzungen zur Pandemie und den getroffenen Maßnahmen - im Vergleich zur Gesamtbevölkerung eine höhere Risikowahrnehmung. Unter anderem empfanden mehr als $40 \%$ der befragten Personen die bestehenden COVID-19-Schutzmaßnahmen in Deutschland zum Zeitpunkt der Befragung als nicht weitgehend genug, bei Infratest dimap (2020a) lag dieser Wert im Oktober 2020 bei $27 \%$ und im November bei $16 \%$ (Infratest dimap 2020b).

\subsection{Veränderung der pflegerischen Versorgung und der Kontaktmöglichkeiten im häuslichen Setting}

\subsubsection{Inanspruchnahme und Veränderung von Unterstützungsleistungen}

Im Zuge des ersten Lockdowns der COVID-19-Pandemie konnte eine Vielzahl von professionellen Unterstützungsleistungen gar nicht oder nur eingeschränkt unter Sicherheitsauflagen angeboten werden. Die Tagespflege konnte lediglich eine Notfallbetreuung für besonders Bedürftige anbieten. Die Inanspruch- nahme der Kurzzeit- und Verhinderungspflege unterlag Quarantäne-Auflagen. Für ambulante Pflegedienste galten Hinweise zu Maßnahmen der Infektionsprävention. Die Vorgaben unterschieden sich länderspezifisch in ihrer Reichund Tragweite.

Die Einschränkungen wirkten auf die Versorgungssituation der Befragten wie folgt (- Tab. 3.3): Von den Pflegehaushalten $(52,9 \%)$, die vor der COVID-19-Pandemie einen Pflegedienst in Anspruch nahmen, gab mehr als ein Viertel $(28,0 \%)$ an, die Inanspruchnahme eingeschränkt oder ganz auf die Leistung verzichten zu haben. Von denen war bei mehr als der Hälfte $(52,9 \%)$ die Entscheidung, die Inanspruchnahme $\mathrm{zu}$ reduzieren oder einzustellen, die gemeinsame Entscheidung zwischen Anbieter und Pflegehaushalt. Die Tagespflege - vor der CoronaPandemie nutzte etwas mehr als jeder zehnte Pflegehaushalt $(11,4 \%)$ diese - konnte von knapp zwei Dritteln $(65,6 \%)$ während des ersten Lockdowns nur eingeschränkt oder gar nicht in Anspruch genommen werden. Bei einem Großteil $(61,7 \%)$ dieser Pflegehaushalte hat der Anbieter entsprechend den rechtlichen Vorgaben während der ersten PandemieWelle die Leistung reduziert bzw. eingestellt. Weitere Entlastungsangebote wie Verhinderungs- oder Kurzzeitpflege, die vor der COVID-19-Pandemie von rund jedem Fünften $(21,3 \%)$ genutzt wurden, wurden ebenso in etwas mehr als der Hälfte der Fälle $(55,0 \%)$ im Lockdown eingeschränkt bzw. eingestellt, bei $58 \%$ auf Wunsch des Pflegehaushalts, bei knapp einem Drittel (30,9\%) durch den Anbieter (- Tab. 3.3).

Neben den professionellen Unterstützungsleistungen sind gleichzeitig auch die Hilfen des informellen Netzwerks in der Pandemie eingebrochen ( $\bullet$ Tab. 3.3). Unterstützung durch Freunde und Bekannte erhielten vor dem ersten Lockdown $43 \%$ der befragten Haushalte, bei $54 \%$ dieser Haushalte wurde die Unterstützung während des Betrachtungszeitraums eingeschränkt oder reduziert. In den meisten Fällen (43,3\%) mit Einschränkungen war es der gemeinsame Wunsch, die Unterstützung zu 
Tab. 3.3 Inanspruchnahme und Veränderung von Unterstützungsleistungen während des Corona-Zeitraums von März bis Mai 2020, häusliches Setting, in \%

\begin{tabular}{|c|c|c|c|c|c|c|c|c|}
\hline \multicolumn{3}{|c|}{$\begin{array}{l}\text { Nutzung vor der COVID-19- } \\
\text { Pandemie }\end{array}$} & \multicolumn{2}{|c|}{$\begin{array}{l}\text { Von diesen haben während } \\
\text { des Corona-Zeitraums } \\
\text { von März bis Mai } 2020 \text { die } \\
\text { Unterstützung ... }\end{array}$} & \multicolumn{4}{|c|}{$\begin{array}{l}\text { Von den Nutzern, die während des Coro- } \\
\text { na-Zeitraums von März bis Mai } 2020 \\
\text { die Inanspruchnahme eingeschränkt/ } \\
\text { eingestellt haben, erfolgte dies... }\end{array}$} \\
\hline & $\%$ & $\mathbf{N}$ & $\begin{array}{l}\text { unverändert } \\
\text { in Anspruch } \\
\text { genommen }\end{array}$ & $\begin{array}{l}\text { verringert } \\
\text { oder ein- } \\
\text { gestellt }\end{array}$ & $\mathbf{N}$ & $\begin{array}{l}\text { auf } \\
\text { Wunsch } \\
\text { des Pflege- } \\
\text { haushalts }\end{array}$ & $\begin{array}{l}\text { durch den } \\
\text { Anbieter/ } \\
\text { die andere } \\
\text { Person }\end{array}$ & $\begin{array}{l}\text { auf } \\
\text { gemein- } \\
\text { samen } \\
\text { Wunsch }\end{array}$ \\
\hline $\begin{array}{l}\text { Pflegedienst } \\
\mathrm{N}=503\end{array}$ & 52,9 & 266 & 69,2 & 28,0 & 74 & 44,1 & 33,0 & 52,9 \\
\hline $\begin{array}{l}\text { Tagespflege } \\
\mathrm{N}=504\end{array}$ & 11,4 & 58 & 27,3 & 65,6 & 37 & 32,1 & 61,7 & 11,4 \\
\hline $\begin{array}{l}\text { Entlastungsangebote } \\
\text { (wie Verhinde- } \\
\text { rungspflege oder } \\
\text { Kurzzeitpflege) } \\
\mathrm{N}=503\end{array}$ & 21,3 & 107 & 39,0 & 55,0 & 58 & 58,7 & 30,9 & 21,3 \\
\hline $\begin{array}{l}\text { Unterstützung durch } \\
\text { Freunde und } \mathrm{Be}- \\
\text { kannte } \\
\mathrm{N}=500\end{array}$ & 43,3 & 217 & 42,9 & 54,0 & 117 & 29,3 & 27,2 & 43,3 \\
\hline $\begin{array}{l}\text { Haushaltshilfe } \\
\mathrm{N}=500\end{array}$ & 54,7 & 274 & 58,9 & 40,2 & 110 & 46,2 & 29,7 & 54,7 \\
\hline
\end{tabular}

Pflege-Report 2021

reduzieren. Ferner nutzten $55 \%$ der befragten Pflegehaushalte vor der Corona-Pandemie eine Haushaltshilfe (Haushaltshilfen, Putzkräfte oder andere bezahlte Hilfen, die im Alltag Unterstützung im Pflegehaushalt der pflegebedürftigen Person leisten), eingeschränkt wurde diese Hilfe bei $40 \%$.

\subsubsection{Veränderung der Kontaktmöglichkeiten}

Zur Beurteilung der Versorgungssituation der betroffenen Pflegehaushalte wurden vertiefend die konkreten Kontakte unter der Pandemiezeit März bis Mai 2020 erfragt. - Abb. 3.5 verdeutlicht die starken Einschränkungen der sozialen Teilhabe für die pflegebedürftigen Personen während des ersten Lockdowns. Laut Angaben der befragten Pflegepersonen haben knapp $80 \%$ der nahestehenden Personen (z. B. Familienmitglieder und Freunde) den Kontakt verglichen mit der Zeit vor dem Lockdown eingestellt oder verringert. Lediglich bei jeder vierten pflegebedürftigen Person (19\%) wurden die Kontakte aufrechterhalten.

Auch ein Großteil der Pflegepersonen hat den eigenen Umgang mit der pflegebedürftigen Person verändert (• Abb. 3.5). Mehr als die Hälfte der Befragten $(52,1 \%)$ hat körperliche Berührungen eingestellt oder verringert. Gleichzeitig haben $68 \%$ der pflegebedürftigen Personen während des Lockdowns ihre Wohnung bzw. ihr Haus seltener oder gar nicht mehr verlassen. Bezüglich des durch die be- 


\section{Während des Corona-Zeitraums von März bis Mai 2020 ...}

haben Familienmitglieder, Freunde oder Nachbarn etc. ihre Besuche bei der pflegebedürftigen Person, verglichen mit der Zeit davor,...N = 499

habe ich körperliche Berührungen mit der pflegebedürftigen Person, wie z.B. durch Handkontakt, Umarmungen oder Küsse, verglichen mit der Zeit davor,...N = 502

habe ich die Zeit, die ich mich um die von mir gepflegte Person kümmere, verglichen mit der Zeit davor,...N = 502 habe ich meinen persönlichen Kontakt zur pflegebedürftigen Person, verglichen mit der Zeit davor,... N = 502

hat die pflegebedürftige Person das Verlassen ihrer Wohnung bzw. ihres Hauses, verglichen mit der Zeit davor, $. . . N=499$

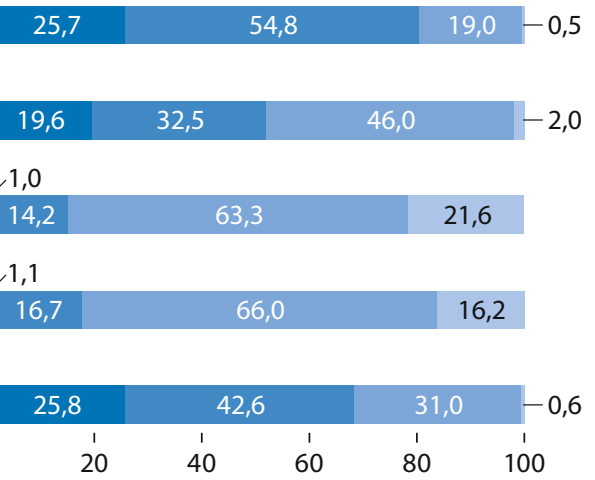

eingestellt verringert nicht verändert intensiviert

- Abb. 3.5 Veränderte Kontaktmöglichkeiten der pflegebedürftigen Person, häusliches Setting, in \%

Durch die getroffenen Maßnahmen ist die Gefahr, dass sich die pflegebedürftige Person mit dem neuartigen Corona-Virus infiziert, deutlich reduziert. $\mathrm{N}=499$

Besonders das Pflegepersonal stellt ein großes Infektionsrisiko dar. $\mathrm{N}=478$ Ich habe Angst, selber die pflegebedürftige
Person zu infizieren. $N=483$

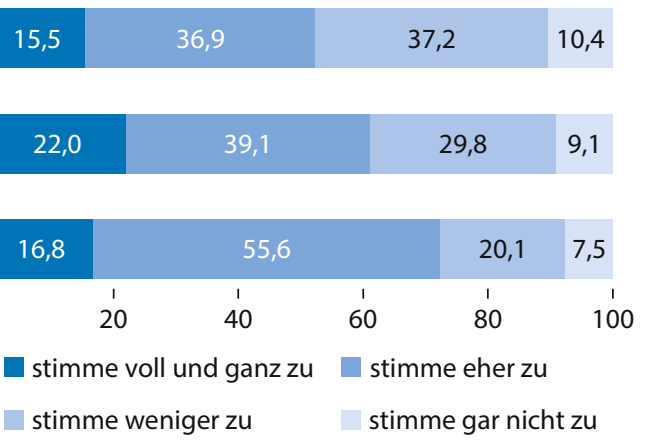

Pflege-Report 2021

- Abb. 3.6 Wahrgenommenes Infektionsrisiko, häusliches Setting, in \%

fragte Person geleisteten Pflegeaufwands zeigte sich vor dem Hintergrund von Kontaktreduktion einerseits und Betreuungsbedarf andererseits ein heterogenes Bild: Bei rund jedem Fünften $(21,6 \%)$ hat sich der Pflegeaufwand intensiviert, gleichzeitig hat sich bei rund $14 \%$ der Pflegeaufwand mindestens verringert (• Abb. 3.5).

Nach Einschätzung von knapp der Hälfte $(52,4 \%)$ der befragten Pflegepersonen redu- zieren die getroffenen Maßnahmen zur Eindämmung der Corona-Pandemie das Infektionsrisiko der pflegebedürftigen Person. GroBe Angst besteht, die pflegebedürftige Person selbst $\mathrm{zu}$ infizieren $(72,4 \%)$. Ferner bewertet ein Großteil der Befragten das Pflegepersonal als großes Infektionsrisiko $(61,1 \%)$ (- Abb. 3.6). 


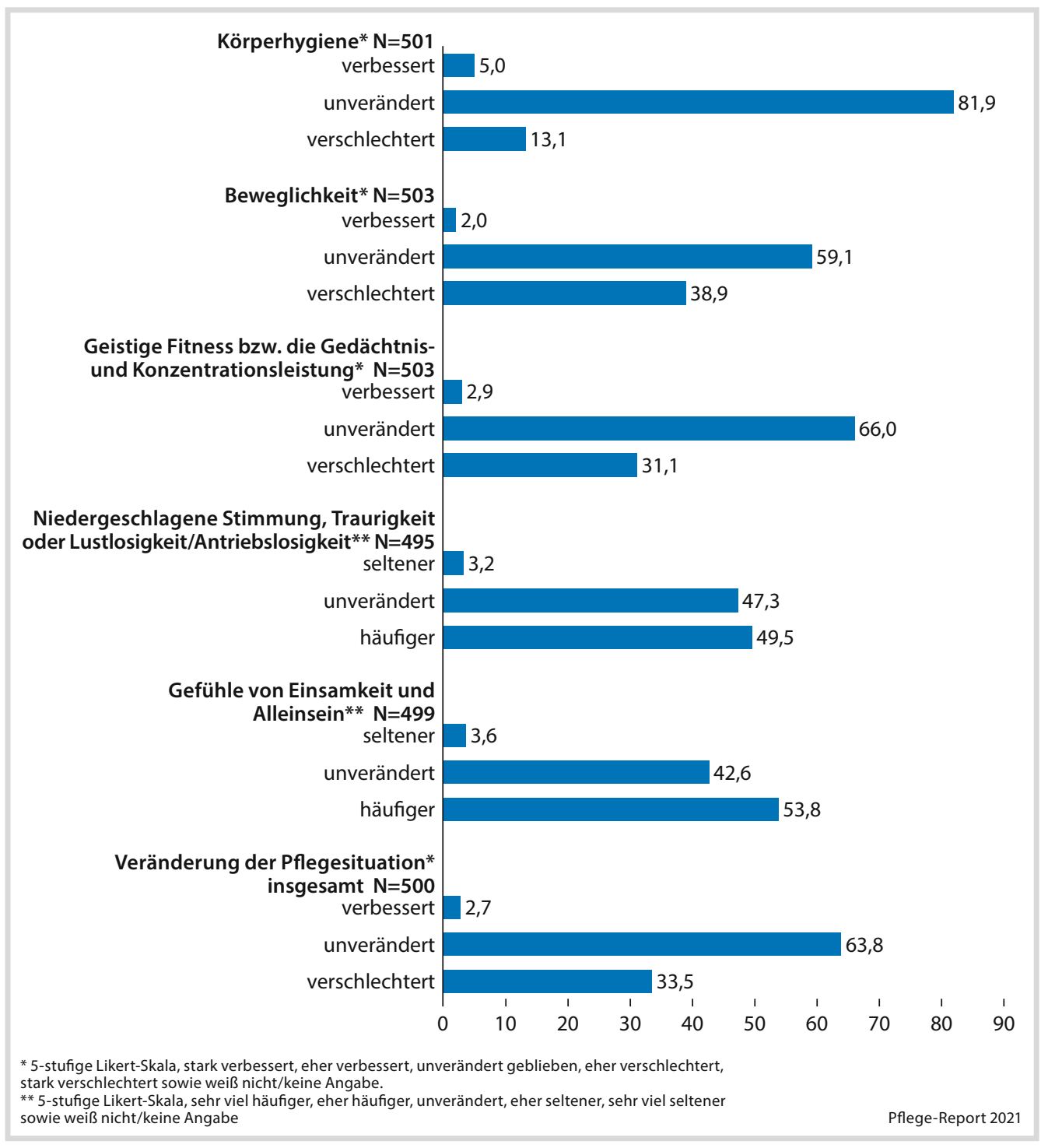

- Abb. 3.7 Wie hat sich der körperliche, geistige und psychische Zustand der pflegebedürftigen Person Ihrer Einschätzung nach unter den Corona-bedingten Einschränkungen im Corona-Zeitraum März bis Mai 2020 verändert? Häusliches Setting, in \%

\subsubsection{Bewertung der pflegerischen Versorgung}

Erhoben wurde außerdem, welchen Einfluss die Corona-bedingt veränderte Versorgungssituation zwischen März bis Mai 2020 aus Sicht der Befragten auf den körperlichen, kognitiven und psychischen Zustand der pflegebedürftigen Person hatte (- Abb. 3.7). Es wird deutlich, dass der Lockdown besonders als Herausforderung für die psychische Gesundheit der pflegebedürftigen Personen wahrgenommen wurde. So berichteten die Pflegepersonen 
die stärksten negativen Veränderungen in den Bereichen geistige Fitness, emotionale Stimmungen sowie Gefühle von Einsamkeit und Alleinsein (• Abb. 3.7). Die geistige Fitness bzw. die Gedächtnis- und Konzentrationsleistung der pflegebedürftigen Person hat sich laut fast einem Drittel $(31,1 \%)$ der befragten Pflegepersonen im Vergleich zu vorher eher oder stark verschlechtert. Auch beobachtete fast die Hälfte $(49,5 \%)$ sehr häufig oder häufiger eine niedergeschlagene Stimmung, Traurigkeit oder Lustlosigkeit bzw. Antriebslosigkeit bei der pflegebedürftigen Person. Sehr häufige oder häufigere Gefühle von Einsamkeit und Alleinsein seitens der pflegebedürftigen Person im Vergleich zu der Zeit vor der Pandemie gaben gut die Hälfte der Befragten $(53,8 \%)$ an. Aber auch die Entwicklung der Beweglichkeit (Gehen, Aufstehen, Treppensteigen etc.) wurde von rund $39 \%$ negativ beurteilt. Eine verschlechterte Körperhygiene der gepflegten Person berichteten hingegen nur $13 \%$ der Pflegepersonen (• Abb. 3.7). In der Gesamtschau geht jeder Dritte Befragte (33,5\%) von einer Verschlechterung der Pflegesituation aus.

\subsubsection{Belastungssituation der Pflegeperson}

Neben den Blick auf die pflegebedürftige Person wurde auch die Belastungssituation der Pflegeperson während des ersten Lockdowns erfragt (- Abb. 3.8). Wenngleich in den meisten Bereichen große Anteile der befragten Pflegepersonen keine Veränderungen wahrgenommen haben - hier schwanken die Werte zwischen $47 \%$ bezüglich ,Gefühlen der Hilflosigkeit“ und $63 \%$ bezüglich „Konflikten mit der pflegebedürftigen Person“ - wurden in allen Kategorien im großen Umfang emotionale Belastungen beschrieben. So berichteten $44 \%$ von häufigeren Gefühlen der Hilfslosigkeit, $39 \%$ von häufigeren Gefühlen von Wut und Angst und $32 \%$ über häufigere Verzweiflungsgefühle. Auch die Konflikte mit der pflegebedürftigen Person nahmen laut $30 \%$ der Pflegepersonen zu.

Ergänzend wurden die Pflegepersonen gefragt, wie sie sich insgesamt bei der Bewältigung der Pflege während des Betrachtungs-

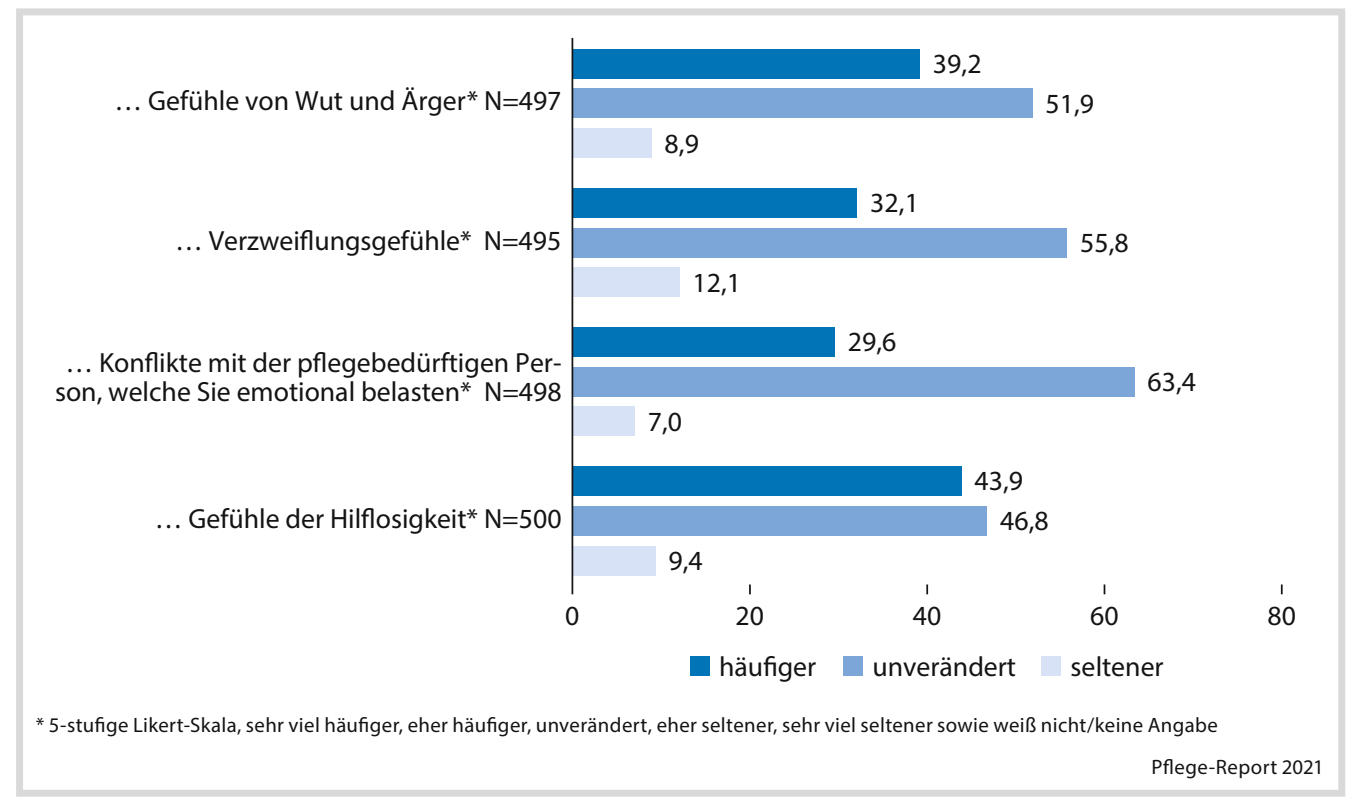

- Abb. 3.8 Emotionaler Zustand der Pflegeperson während des Lockdowns. Unter den Corona-bedingten Einschränkungen im Corona-Zeitraum März bis Mai 2020 hatten Sie selbst im Vergleich zu vorher ... ? Häusliches Setting, in \% 


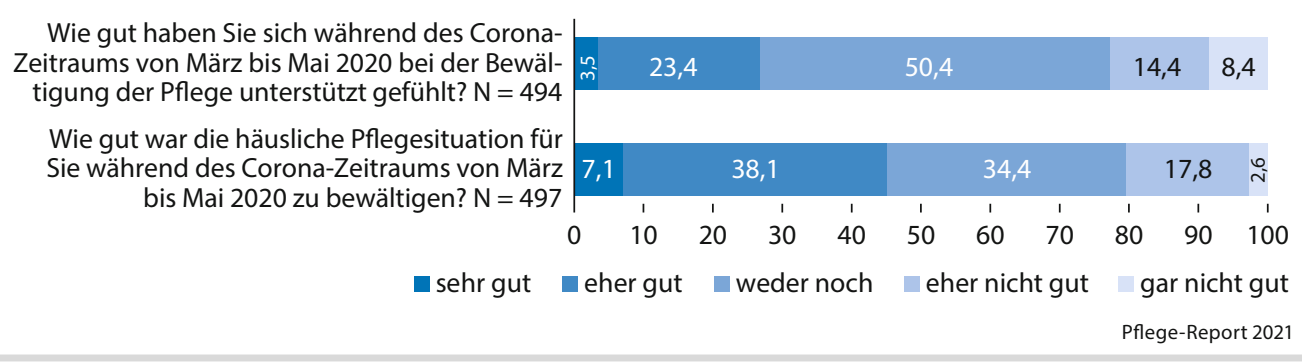

- Abb. 3.9 Unterstützungsbedarfe und Bewältigung der Pflegesituation, häusliches Setting, in \%

zeitraums unterstützt fühlten: Etwas mehr als jeder fünfte Befragte $(22,8 \%)$ fühlte sich eher nicht oder gar nicht gut unterstützt, demgegenüber aber auch $27 \%$ eher gut bis sehr gut (- Abb. 3.9). Verglichen mit der WIdO-Befragung von 2019 und somit genau ein Jahr vor der hiesigen Befragung haben sich diese Werte eher verschlechtert: Damals gaben $45 \%$ an, sich gut oder sehr gut unterstützt zu fühlen. Der Anteil an Befragten, die sich eher oder überhaupt nicht gut unterstützt fühlte, war mit $22 \%$ jedoch ähnlich hoch (Räker et al. 2020). Zusammenfassend gab jede fünfte Pflegeperson $(20,4 \%)$ an, die Pflegesituation nicht gut oder gar nicht gut bewältigen zu können, auch dieser Anteil lag 2019 in einem ähnlichen Bereich (ebd.).

\subsubsection{Zusammenfassung und Zwischenfazit zur pflegerischen Versorgung im häuslichen Setting}

Während des ersten Lockdowns unterlagen die Pflegehaushalte und somit die pflegebedürftigen Personen im ambulanten Setting starken Einschränkungen. Neben den professionellen Unterstützungsleistungen sind gleichzeitig auch die Hilfen durch das informelle Netzwerk in der Pandemie eingebrochen. Damit einhergehend waren die Möglichkeiten zur sozialen Teilhabe stark eingeschränkt; so zeigte sich, dass die pflegebedürftigen Per- sonen auf Kontakt zu Menschen außerhalb des Pflegehaushalts abgesehen von der Pflegeperson weitgehend verzichten mussten. So verwundert es nicht, dass in der Gesamtschau jeder dritte Befragte $(33,5 \%)$ von einer Verschlechterung der Pflegesituation ausgeht (- Abb. 3.7). Zu beachten gilt jedoch, dass für alle Fragenbereiche ein heterogenes Bild zu verzeichnen ist und es Teilgruppen gibt, die stärker als andere von den Auswirkungen der Pandemie betroffen zu sein scheinen. Die Ergebnisse decken sich mit den Befragungen von Horn und Schweppe (2020) sowie Eggert et al. (2020) zur ersten COVID-19-Welle. Gleichwohl ist der Vergleich eingeschränkt, da davon auszugehen ist, dass der konkrete Erhebungszeitpunkt einen relativ starken Einfluss auf das Antwortverhalten hat. Dennoch: Auch in diesen Studien werden die starken Einschränkungen bei der Unterstützung durch Angehörige, Freunde und Nachbarn aufgezeigt und auch dort geht jeder Dritte Befragte (33,5\%, Eggert et al. 2020) bzw. sogar die Hälfte der Befragten (50\%, Horn und Schweppe 2020) von einer Verschlechterung der Pflegesituation aus.

Mit Blick auf die Befragten - also die Pflegepersonen selbst - wird deutlich, dass der Lockdown ebenso besondere Herausforderungen birgt. Für alle abgefragten Kategorien berichten die befragten Personen in großem Umfang emotionale Belastungen. Am häufigsten wird dabei das Gefühl der Hilflosigkeit genannt. Jeder fünfte Pflegehaushalt fühlte sich in der Pandemie nicht gut unterstützt oder gibt an, die Pflege nicht gut bewältigen zu kön- 
nen. Die Ergebnisse weisen wiederum in die gleiche Richtung wie bei Eggert et al. (2020) und Horn und Schweppe (2020). Dort sagen $25 \%$ bzw. sogar $38 \%$, dass sie mit der Pflegesituation (eher oder voll und ganz) überfordert seien. Hervorzuheben ist jedoch auch, dass der in der hiesigen Befragung erfasste Anteil der Haushalte, der angegeben hat, die häusliche Pflegesituation nicht bewältigen zu können, im Vergleich zur 2019 durchgeführten WIdO-Online-Panel-Befragung eher konstant geblieben ist. Schlussfolgerungen hieraus sind jedoch nur bedingt möglich, da es sich nicht um eine Querschnittbefragung, d. h. eine Befragung der gleichen Personen im zeitlichen Verlauf, handelt und die Stichprobenzusammensetzungen ferner leicht variieren. Eine These könnte sein, dass funktionierende Pflegearrangements während der ersten Welle mit hinreichenden Bewältigungsressourcen verbunden waren und die Pflegepersonen insofern auch in der Krise die Situation als für sich beherrschbar empfanden - trotz der erheblichen Einschränkungen des Unterstützungsnetzwerks. Wie sich diese Einschätzung im Laufe der Pandemie verändert und wann die Coping-Ressourcen erschöpft sind, bleibt insofern zu untersuchen. Ferner ist zu vermuten, dass sich die Antworten auf die Frage auf die persönliche Pflegebeziehung respektive -situation beziehen, wobei die verschärften Rahmenbedingungen der COVID-19-Pandemie weitgehend ausgeblendet oder als schlicht gegeben betrachtet wurden.

\subsection{Veränderung der pflegerischen Versorgung und der Kontaktmöglichkeiten im vollstationären Setting}

\subsubsection{Veränderung der Kontaktmöglichkeiten}

Anders als die Teilstichprobe der ambulant versorgten Pflegebedürftigen wurden die Bezugspersonen von vollstationär Pflegebedürftigen weniger nach dem Versorgungsmix als vielmehr zu den veränderten Kontaktmöglichkeiten des Pflegebedürftigen befragt. Mit Blick auf die Möglichkeiten zur sozialen Teilhabe und Aktivität wird deutlich, dass ein Großteil der pflegebedürftigen Personen drastische Einschränkungen in Kauf nehmen musste. $43 \%$ der befragten Bezugspersonen berichten, dass zwischen März und Mai 2020 die Möglichkeit zum persönlichem Kontakt, auch unter Einhaltung von Schutzmaßnahmen, gar nicht gegeben war. Für ein weiteres knappes Drittel $(30,3 \%)$ war diese Möglichkeit nur selten gegeben (-Abb. 3.10). Telefonischer Kontakt war demgegenüber für zwei Drittel $(71,5 \%)$ der Bezugspersonen oft oder uneingeschränkt möglich. Rund jeder achten Bezugsperson $(12,5 \%)$ war es oft oder uneingeschränkt möglich, während des ersten Lockdowns mit der pflegebedürftigen Person mithilfe eines Tablets zu kommunizieren. Eine zusammengefasste Betrachtung der Fragen ergibt, dass $30 \%$ der Befragten sowohl bei der Frage, ob telefonischer Kontakt möglich war als auch bei der Frage, ob Kontakt via Tablet möglich war, „gar nicht“ oder ,selten“ angaben und somit nur eingeschränkt mit der pflegebedürftigen Person kommunizieren konnten.

Nach Angaben der Bezugspersonen war es $16 \%$ der pflegebedürftigen Personen ferner nicht möglich, das eigene Zimmer zu verlassen, weiteren $25 \%$ war es nur selten möglich. Lediglich für $36 \%$ war es uneingeschränkt möglich (•Abb. 3.10). Erfragt worden war 
Während des Corona-Zeitraums von März bis Mai 2020 bestand die Möglichkeit...

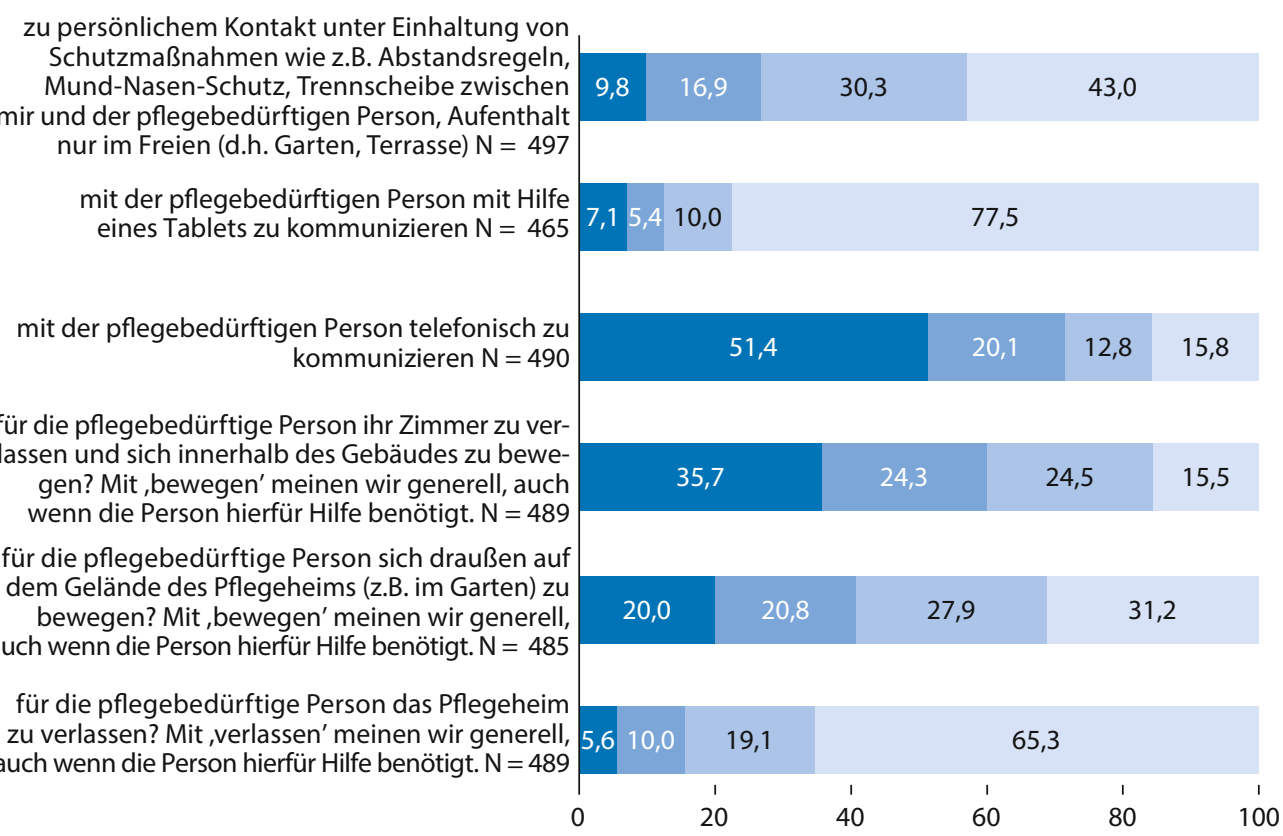

war uneingeschränkt gegeben.

war oft gegeben.

war gar nicht gegeben.

Pflege-Report 2021

- Abb. 3.10 Kontakt- und Mobilitätsmöglichkeiten der pflegebedürftigen Person während des Corona-Zeitraums von März bis Mai 2020, vollstationäres Setting, in \%

auch, wie mobil die Bewohnenden vor der Pandemiesituation waren. $36 \%$ der pflegebedürftigen Personen, die vor der Pandemie täglich oder mehrmals in der Woche das Zimmer verlassen haben $(84,3 \%)$, hatten diese Möglichkeit während des ersten Lockdowns gar nicht oder nur selten.

Die Pflegeeinrichtung allein oder mit Hilfe $\mathrm{zu}$ verlassen war lediglich für rund $16 \%$ der pflegebedürftigen Personen oft oder uneingeschränkt möglich. Es wird deutlich, dass die Möglichkeiten zur Mobilität deutlich eingeschränkt wurden: Vor der COVID-19-Pandemie hatten $29 \%$ der pflegebedürftigen Personen die Pflegeeinrichtung täglich oder mehrmals in der Woche verlassen, für $76 \%$ dieser pflegebedürftigen Personen war dies während des ersten Lockdowns gar nicht oder nur selten möglich.
Sich zumindest auf dem Gelände der Pflegeeinrichtung (z.B. im Garten) allein oder mit Hilfe bewegen zu können, war für mehr als die Hälfte der pflegebedürftigen Personen $(59,1 \%)$ gar nicht oder selten gegeben (- Abb. 3.10).

Die Bezugspersonen wurden im Anschluss gefragt, ob sie die getroffenen Maßnahmen als wirksamen Schutz zur Reduzierung des Infektionsrisikos der pflegebedürftigen Person bewerteten (- Abb. 3.11). $86 \%$ der befragten Bezugspersonen stimmten dieser Aussage zu. Ein Großteil der Befragten $(72,4 \%)$ sieht im Pflegepersonal ein großes Infektionsrisiko, aber auch sich selbst sehen die Befragten als Infektionsrisiko: Fast die Hälfte der befragten Bezugspersonen (46,7\%) berichtete von der Angst, selbst die pflegebedürftige Person zu infizieren. 


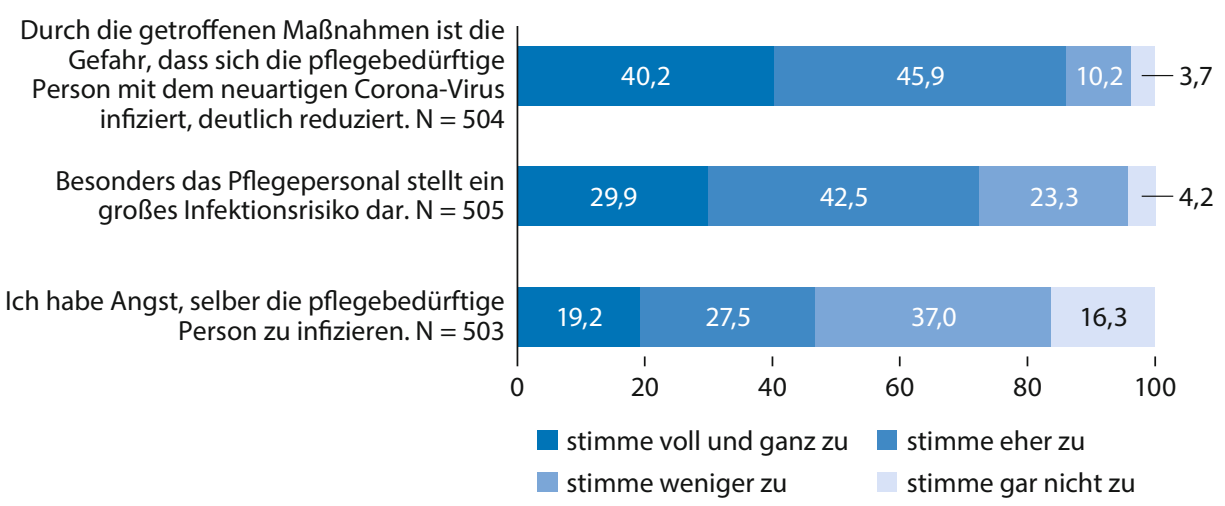

Pflege-Report 2021

- Abb. 3.11 Wahrgenommenes Infektionsrisiko, vollstationäres Setting, in \%

\subsubsection{Bewertung der pflegerischen Versorgung}

Analog zur Befragung der Pflegepersonen im ambulanten Setting wurden auch die Bezugspersonen von Pflegebedürftigen im vollstationären Setting zu den Veränderungen des körperlichen, geistigen und psychischen Zustands der pflegebedürftigen Person durch die Corona-bedingten Einschränkungen im Zeitraum März bis Mai 2020 befragt (- Abb. 3.12).

In allen abgefragten Bereichen wurden deutliche negative Veränderungen wahrgenommen. Außer für die Kategorien „Veränderung der Pflegesituation insgesamt" (42,9\%) sowie „Körperhygiene“ (23,4\%) lagen für alle Bereiche die Anteile der Befragten, die eine Verschlechterung berichteten, sogar über $50 \%$.
Häufigere Gefühle von Einsamkeit und Alleinsein seitens der pflegebedürftigen Person berichteten $71 \%$. Häufigeres Auftreten von niedergeschlagener Stimmung, Traurigkeit oder Lustlosigkeit bzw. Antriebslosigkeit gaben $68 \%$ an. Auch Verschlechterungen der geistigen Fitness bzw. der Gedächtnisund Konzentrationsleistung der pflegebedürftigen Person (61,4\%) sowie ihrer Beweglichkeit (Gehen, Aufstehen, Treppensteigen etc.) $(55,8 \%)$ im Vergleich $\mathrm{zu}$ vorher wurden oft angegeben. Insgesamt zeigt sich vor dem Hintergrund der ergriffenen scharfen Isolationsmaßnahmen in den Pflegeheimen in der ersten Pandemiewelle ein besonders dramatisches Bild der physischen und psychischen Folgen: Für die vollstationär Pflegebedürftigen führten die Maßnahmen ausnahmslos in allen erfassten Kategorien häufiger zu einer Verschlechterung, als dies für den ambulanten Kontext berichtet wird (• Abb. 3.7). 


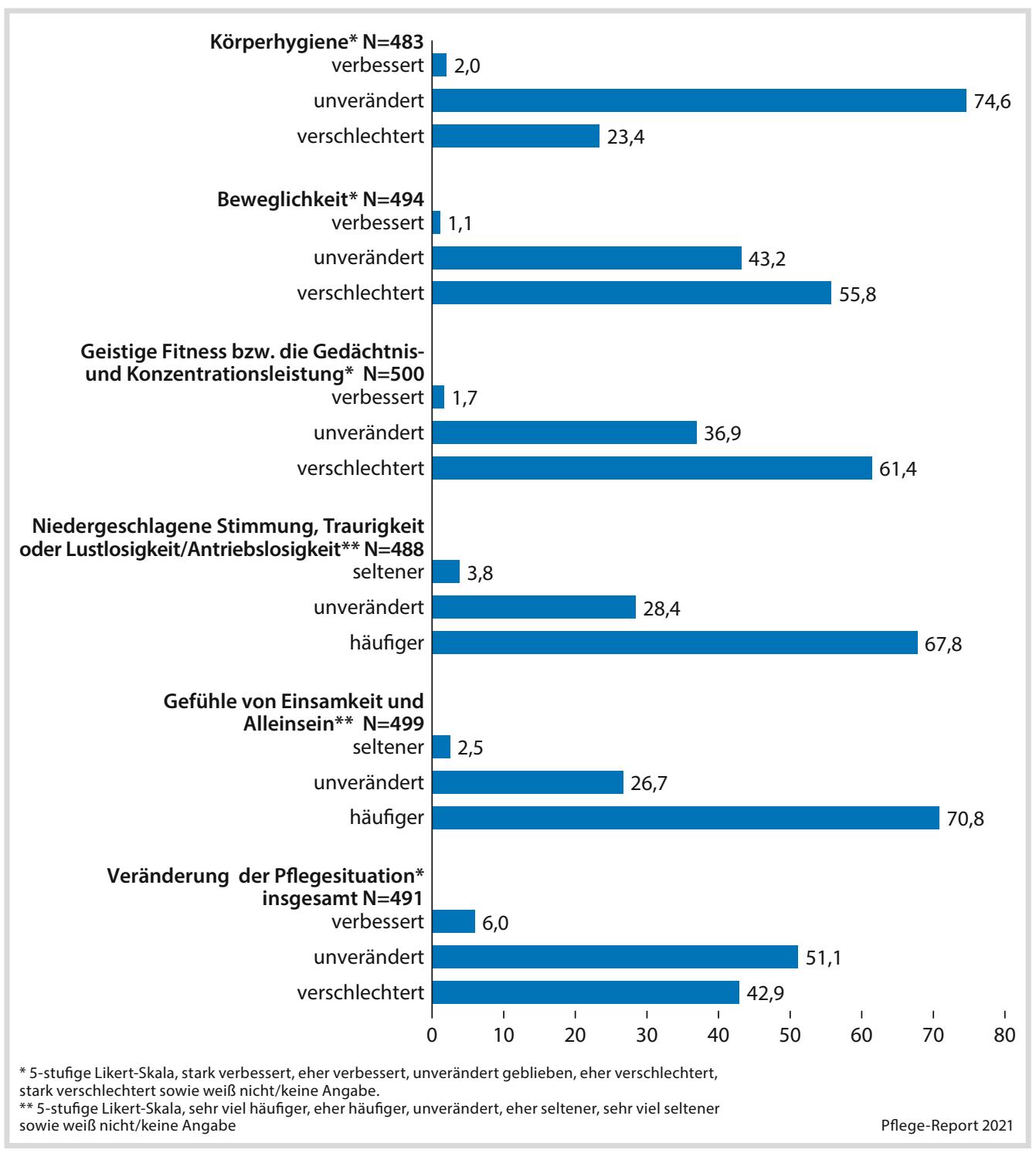

- Abb. 3.12 Wie hat sich der körperliche, geistige und psychische Zustand der pflegebedürftigen Person Ihrer Einschätzung nach unter den Corona-bedingten Einschränkungen im Corona-Zeitraum März bis Mai 2020 verändert? Vollstationäres Setting, in \%

\subsubsection{Belastungssituation der Bezugsperson}

Ergänzend zur wahrgenommenen Veränderung des Zustands der pflegebedürftigen Person wurde auch nach den (emotionalen) Belas- tungen der Bezugsperson während des ersten Lockdowns gefragt (-Abb. 3.13). Besonders das Gefühl von Hilflosigkeit ist aus Sicht der Bezugsperson mit $63 \%$ häufiger im Vergleich zu der Zeit vor der COVID-19-Pandemie aufgetreten. Rund $40 \%$ gaben häufigere Gefühle von Verzweiflung sowie von Wut und Ärger 


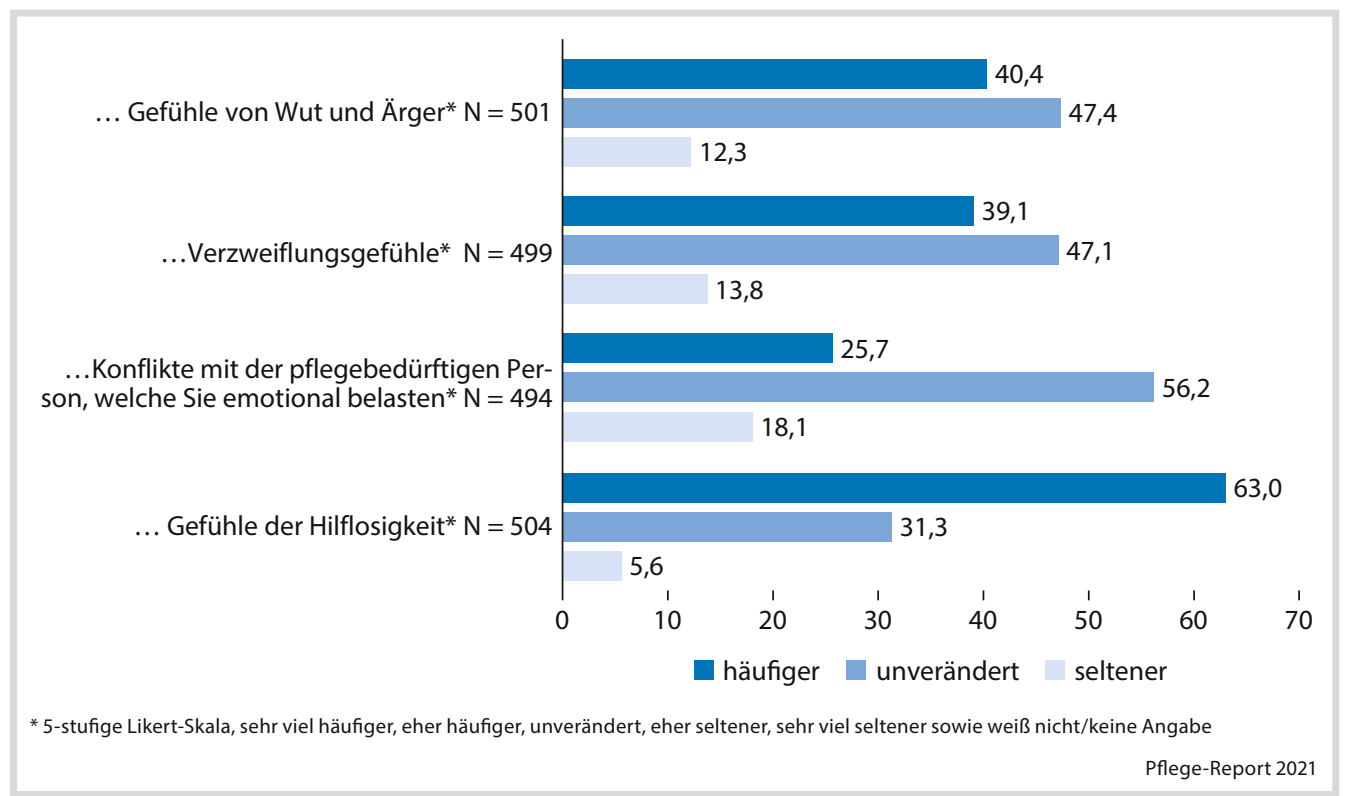

- Abb. 3.13 Emotionaler Zustand der Bezugsperson während des Lockdowns. Unter den Corona-bedingten Einschränkungen im Corona-Zeitraum März bis Mai 2020 hatten Sie selbst im Vergleich zu vorher ... ? Vollstationäres Setting, in $\%$

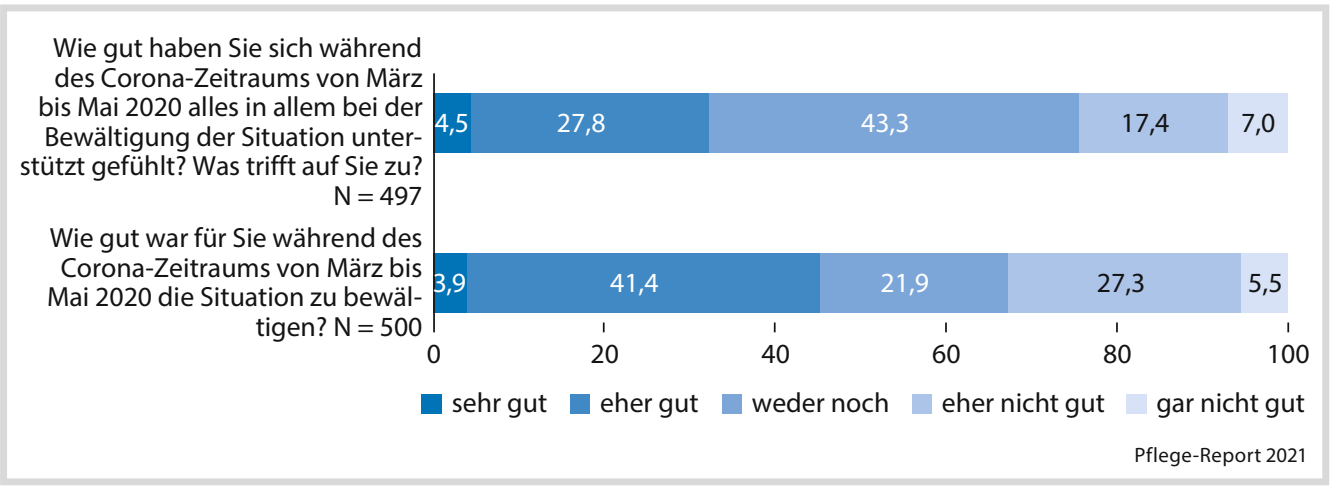

- Abb. 3.14 Wahrgenommene Belastung und Unterstützung der Pflegeperson, vollstationäres Setting, in \%

an. Im Vergleich zur Situation im ambulanten Setting (- Abb. 3.8) wird deutlich, dass Konflikte mit der pflegebedürftigen Person nicht im gleichen Maße in der Pandemie Bedeutung haben, jedoch für das vollstationäre Setting prägend zu sein scheint, dass das Gefühl der Hilfslosigkeit zugenommen hat - dies ist mit
Blick auf die besonders stark eingeschränkten Kontaktmöglichkeiten naheliegend.

Neben dem emotionalen Zustand wurde auch für die Bezugspersonen der vollstationär Pflegebedürftigen die wahrgenommene Belastung erfasst (- Abb. 3.14). Jede vierte Person $(24,4 \%)$ gab an, sich nicht gut unterstützt ge- 
fühlt zu haben, während ein Drittel $(32,3 \%)$ sich gut unterstützt fühlte. Ein weiteres Dritte $(32,8 \%)$ sagte aber auch, dass die Pflegesituation eher nicht oder gar nicht gut zu bewältigen war. Im Vergleich zum ambulanten Setting hier hat jeweils rund jede fünfte Pflegeperson angegeben, sich nicht gut unterstützt zu fühlen respektive mit der Pflegesituation nicht gut zurecht zu kommen (• Abb. 3.9) - überraschen diese Aussagen. $\mathrm{Zu}$ diskutieren ist, inwiefern dies darauf hindeutet, dass die emotionale Belastung aufgrund der fehlenden Kontaktmöglichkeiten höher wiegt als die Belastungen, die aufgrund des Wegbrechens der Unterstützungsnetzwerke zu vermuten sind, Nähe aber dennoch erlauben.

\subsubsection{Fokus ärztliche Versorgung in Pflegeeinrichtungen}

\section{- Veränderung der ärztlichen Versorgung während des ersten Lockdowns}

Neben der Betrachtung der pflegerischen Versorgung und deren Veränderung aufgrund der COVID-19-Pandemie wurde für das Pflegeheim auch die ärztliche Versorgung in den Blick genommen. Bei etwas mehr als jeder fünften $(22,5 \%)$ pflegebedürftigen Person sind nach Angaben der Bezugsperson alle bereits vereinbarte Termine mit dem Hausarzt oder der Hausärztin während der Lockdown-Phase März bis Mai 2020 ausgefallen, bei weiteren $43 \%$ haben Termine nur teilweise stattgefunden. Bei Bewohnenden mit vereinbarter fachärztlicher Versorgung haben nur bei gut einem Viertel der Personen alle Termine stattgefunden $(27,4 \%)(\bullet$ Abb. 3.15).
Die Bezugspersonen wurden des Weiteren zum Zugang der Bewohnenden zur haus- und fachärztlichen Versorgung verglichen mit der Zeit davor befragt (- Abb. 3.16). Etwas mehr als jede vierte befragte Person $(26,7 \%)$ gab an, dass sich die allgemeine Erreichbarkeit von Hausarztpraxen verschlechtert habe. Konkret wurde die Möglichkeit, bei Bedarf einen Termin bei dem Hausarzt oder der Hausärztin zu erhalten, von einem Drittel $(37,4 \%)$ als stark oder eher verschlechtert wahrgenommen. Verschlechterungen bei der Möglichkeit, Rezepte und andere Bescheinigungen durch den Hausarzt bzw. die Hausärztin zu erhalten, berichten $10 \%$.

Die allgemeine Erreichbarkeit eines Facharztes oder einer Fachärztin wurde zwar eher als unverändert wahrgenommen, eine Verschlechterung bei den Terminen gab jedoch fast die Hälfte $(46,1 \%)$ der Befragten an. Ebenso gab jede fünfte befragte Person $(19,7 \%)$ an, dass sich der Erhalt von Rezepten und andere Bescheinigungen verschlechtert habe - dies sind doppelt so viele wie im hausärztlichen Kontext. Hinzuweisen ist darauf, dass es für alle erhobenen Kontaktmöglichkeiten auch Befragte gab, die die Situation im Lockdown positiver als sonst wahrnahmen (- Abb. 3.16).

Die Befragten, die mindestens einen abgesagten Termin zur hausärztlichen und fachärztlichen Versorgung angegeben haben, konnten mehrere Initiatoren für die Terminabsage benennen. - Tab. 3.4 zeigt den Anteil, den die jeweiligen Initiatoren der Terminabsagen für beide Versorgungsbereiche haben. Deutlich wird, dass die Absage der Termine am häufigsten durch die Pflegeeinrichtungen erfolgte. Erst an zweiter Stelle wurden die Ärzte und Ärztinnen als Initiatoren benannt. Dass die Absage durch sie selbst oder die Pflegebedürftigen entstand, gaben die Befragten deutlich seltener an. 

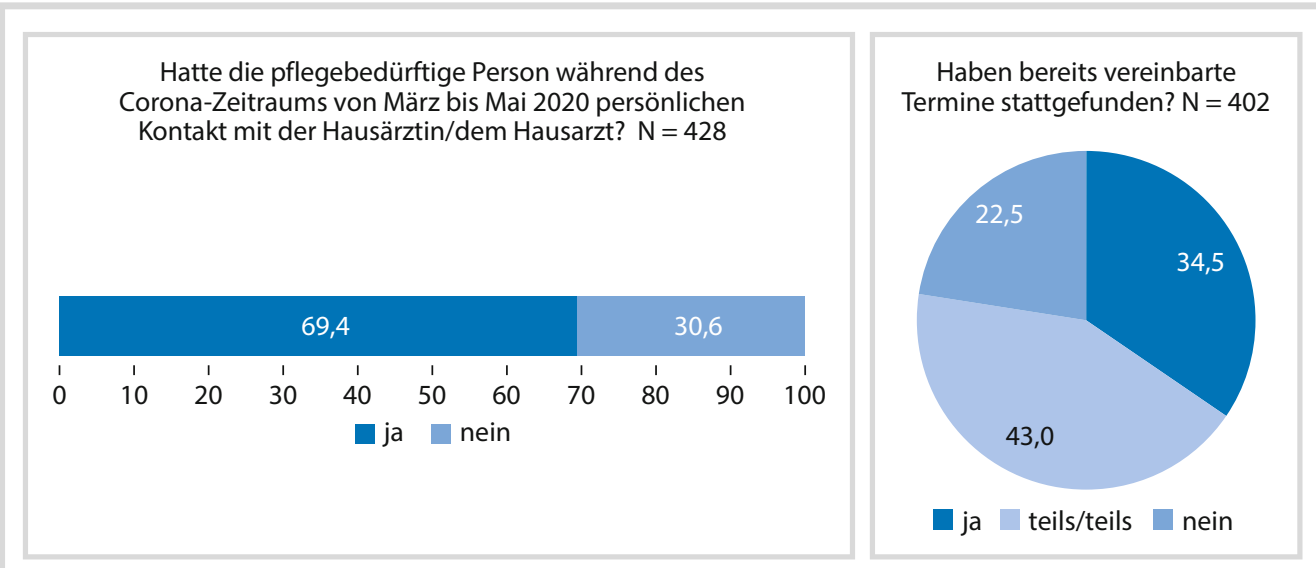

Hatte die pflegebedürftige Person während des Corona-Zeitraums von März bis Mai 2020 persönlichen Kontakt mit einer Fachärztin/einem Facharzt $\mathrm{N}=440$

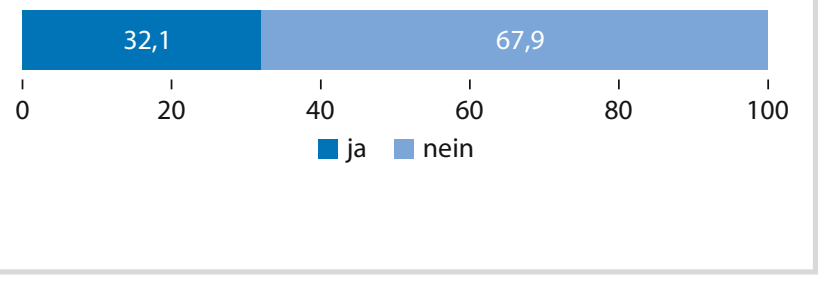

Haben bereits vereinbarte Termine stattgefunden? N = 309

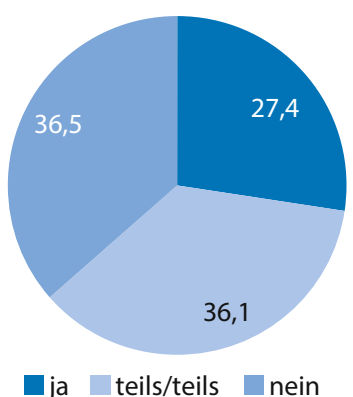

ja teils/teils nein

Pflege-Report 2021

- Abb. 3.15 Termine zur haus- und fachärztlichen Versorgung während des Corona-Zeitraums von März bis Mai 2020, vollstationäres Setting, in \%

- Tab. 3.4 Durch wen wurden bereits vereinbarte Termine abgesagt?

\begin{tabular}{|c|c|c|}
\hline & $\begin{array}{l}\text { Hausärztliche Versorgung (min- } \\
\text { destens ein ausgefallener Termin } \\
(\mathrm{N}=\mathbf{2 6 3}) \text { ) }\end{array}$ & $\begin{array}{l}\text { Fachärztliche Versorgung (min- } \\
\text { destens ein ausgefallener Termin } \\
(\mathrm{N}=\mathbf{2 2 5}) \text { ) }\end{array}$ \\
\hline & \multicolumn{2}{|c|}{ Anteil der Initiatoren der Terminabsagen ${ }^{\mathrm{a}}$ in \% } \\
\hline Durch den Arzt/die Ärztin & $25,7(n=68)$ & $28,7(n=64)$ \\
\hline $\begin{array}{l}\text { Durch die Bezugsperson/die } \\
\text { pflegebedürftige Person selbst }\end{array}$ & $12,4(n=33)$ & $20,4(n=46)$ \\
\hline Durch die Pflegeeinrichtung & $43,6(n=115)$ & $41,1(n=92)$ \\
\hline Weiß nicht/keine Angabe & $27,0(n=71)$ & $19,2(n=43)$ \\
\hline
\end{tabular}




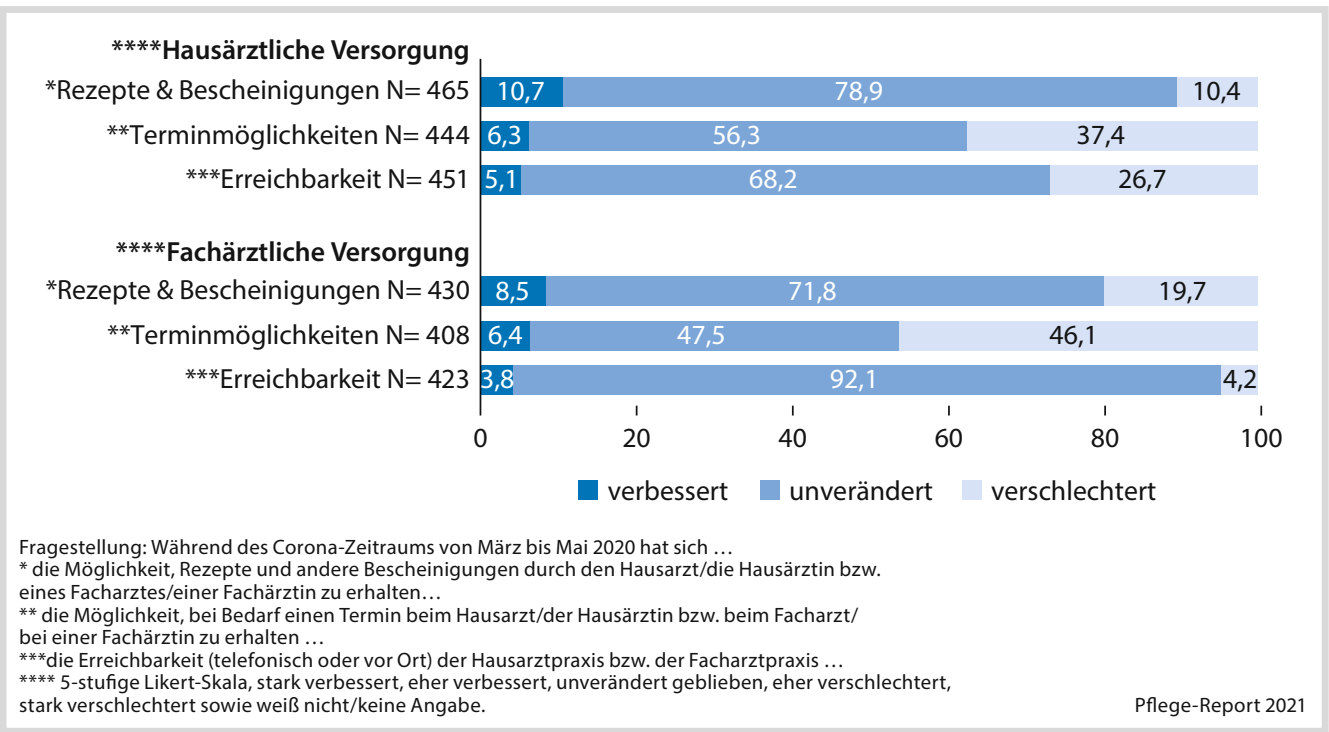

- Abb. 3.16 Veränderung des Zugangs zur gesundheitlichen Versorgung während des Corona-Zeitraums von März bis Mai 2020, vollstationäres Setting, in \%

\section{- Bewertung der veränderten gesundheitlichen Versorgung}

Ergänzend wurden die Bezugspersonen zu ihrer Wahrnehmung der ärztlichen Betreuung des/der Pflegebedürftigen befragt (- Abb. 3.17). Demnach gaben deutlich mehr als ein Drittel (39\%) der befragten Bezugspersonen an, dass während des ersten Lockdowns wichtige Arzttermine nicht erfolgten. Rund jede siebte Bezugsperson (13,8\%) berichtete, dass in dem betrachteten Zeitraum aufgrund fehlender Erreichbarkeit der Arztpraxen auf wichtige Rezepte verzichtet werden muss-
Während des Corona-Zeitraums von März bis Mai 2020 musste auf wichtige Arzttermine (Diagnostik,Untersuchungen, Eingriffe etc.) verzichtet werden. $\mathrm{N}=442$

Durch die fehlende Erreichbarkeit der Arztpraxen während des Corona-Zeitraums von März bis Mai 2020 hat die pflegebedürftige Person wichtige Rezepte (Medikamente, Physiotherapie etc.) nicht erhalten. $\mathrm{N}=461$

Während des Corona-Zeitraums von März bis Mai 2020 bestand aufgrund der Ansteckungsgefahr Angst, persönlich die Arztpraxis aufzusuchen. $\mathrm{N}=460$

stimme voll und ganz zu
11,4

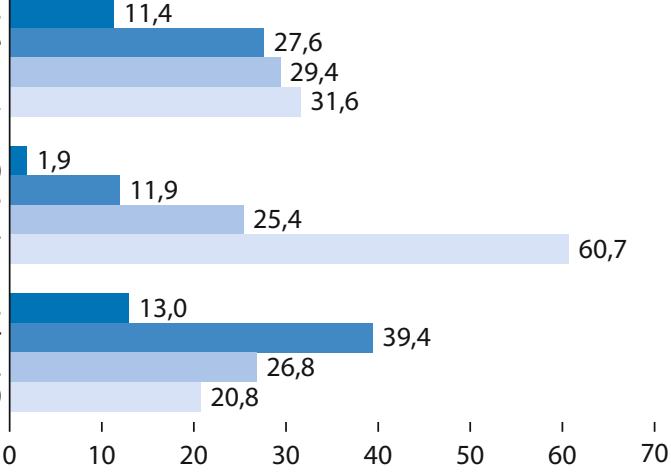


6,6

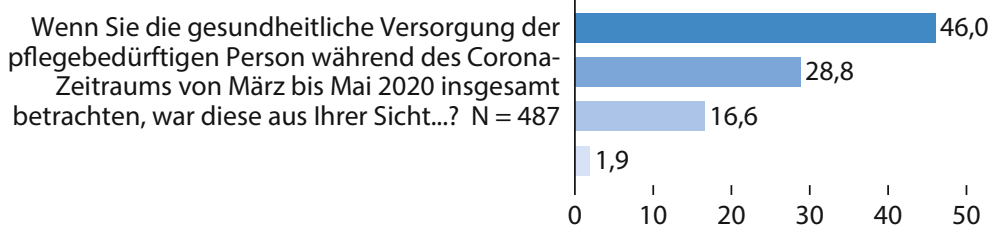

sehrgut ehergut weder noch eher nicht gut gar nicht gut

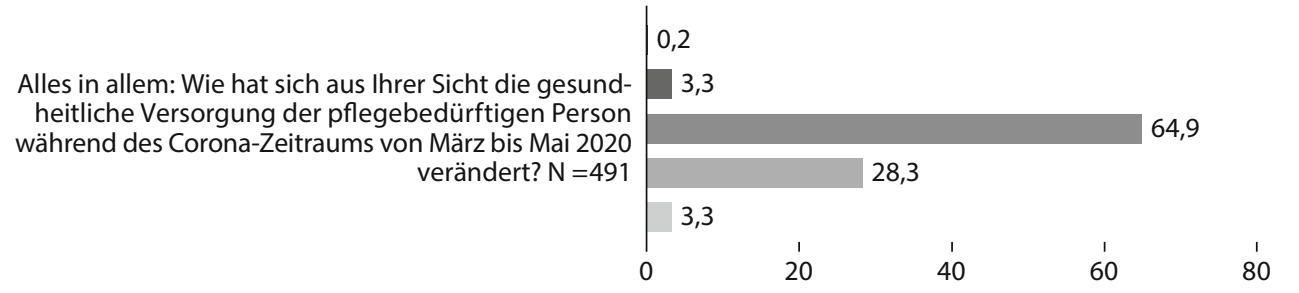

hat sich stark verbessert $\quad$ hat sich eher verbessert

ist unverändert geblieben

hat sich eher verschlechtert hat sich stark verschlechtert

Pflege-Report 2021

- Abb.3.18 Bewertung der gesundheitlichen Versorgung der pflegebedürftigen Person während des Corona-Zeitraums von März bis Mai 2020 und dessen Veränderung, vollstationäres Setting, in \%

te. Gleichzeitig bestanden bei mehr als der Hälfte der Befragten $(52,4 \%)$ aufgrund der Ansteckungsgefahr Ängste, persönlich in die Arztpraxis zu kommen.

Befragt nach der gesundheitlichen Versorgung der pflegebedürftigen Person während der ersten Pandemiewelle gibt etwas mehr als die Hälfte $(52,6 \%)$ der Bezugspersonen in der Gesamtschau eine positive Bewertung (- Abb. 3.18) ab. Andererseits bewertet aber auch jede fünfte Bezugsperson (18,5\%) die Situation als nicht gut. Ferner wird das positive Bild etwas relativiert, wenn man die Situation mit der Lage vor der Corona-Pandemie vergleicht. Diesbezüglich sagt jeder dritte Befragte $(31,6 \%)$, dass sich die gesundheitliche Versorgung verschlechtert habe (• Abb. 3.18).

\subsubsection{Zusammenfassung und Zwischenfazit zur pflegerischen Versorgung im vollstationären Setting}

Drastische Einschnitte erlebten die pflegebedürftigen Personen im vollstationären Versorgungssetting im Bereich der sozialen Teilhabe: Für $43 \%$ der Befragten war ein persönlicher Kontakt zu ihren pflegebedürftigen Angehörigen zwischen März und Mai 2020 nicht möglich, für weitere $30 \%$ nur selten. Jeder Dritte konnte weder telefonisch noch internetbasiert mit dem Angehörigen kommunizieren. Neben dem Kontakt zur Bezugsperson wurde für die Pflegebedürftigen auch die Mobilität in der Form, das eigene Zimmer oder die Pflegeeinrichtung verlassen zu können, stark eingeschränkt. Nicht verwunderlich ist, dass insofern die Mehrheit der befragten Bezugspersonen über deutliche Verschlechterungen des pflegerischen Gesamtzustandes der An- 
gehörigen berichtet. Hervorzuheben ist, dass die Bezugspersonen von vollstationär Pflegebedürftigen ausnahmslos für alle der erfassten Kategorien - Körperhygiene, Beweglichkeit, geistige Fitness, Stimmungslage, Einsamkeit - sowie mit Blick auf die Gesamtsituation häufiger eine Verschlechterung der Situation angeben, als dies die befragten Hauptpflegepersonen für die ambulant Pflegebedürftigen benennen. Bezüglich der gesundheitlichen respektive ärztlichen Versorgung der Pflegeheimbewohnenden sieht ferner rund ein Drittel eine Verschlechterung. Bei der Interpretation ist gleichwohl zu beachten, dass es sich um Fremdeinschätzungen durch Personen handelt, die zum Teil auch vor der Pandemie weniger als einmal pro Woche Kontakt zum Pflegebedürftigen hatten. Gleichwohl decken sich die Ergebnisse von der Tendenz her mit der Befragung von Eggert und Teubner (2021). Hier gaben beispielsweise $74 \%$ der befragten Verantwortlichen aus der Langzeitpflege an, dass sich „Stimmung und Lebensfreude“ bei Bewohnenden ohne Demenz im April 2020 Corona-bedingt eher oder stark verschlechtert haben, bei Bewohnenden mit Demenz lag dieser Wert bei $53 \%$. Ferner bestätigen Horn und Schweppe (2020) wie auch Eggert und Teubner (2021) starke Einschnitte in der ärztlichen Versorgung und explizit den Ausfall von Arztterminen.

Mit Blick auf die Situation der Befragten - also der Bezugsperson selbst - sind überraschend hohe Belastungswerte zu konstatierten: Zwei von drei Befragten geben an, dass sie sich häufiger hilflos fühlen als vor der Pandemie. Gleichzeitig sagt jeder Dritte - im ambulanten Setting spricht hiervon lediglich jeder Fünfte -, dass die Pflegesituation nicht gut zu bewältigen war. Dies kann auf die hohe emotionale Belastung hinweisen, die aus den erheblichen Kontaktbeschränkungen resultiert.

\subsection{Zusammenfassung und Fazit}

Im Rahmen der COVID-19-Pandemie gelten pflegebedürftige Menschen als besonders vulnerabel und anfällig für schwere Krankheitsverläufe. In der Folge zielten und zielen viele Maßnahmen zur Eindämmung der COVID-19-Pandemie auf den Schutz dieser Bevölkerungsgruppe. Die hier vorgelegte Studie unterstreicht, dass es in der häuslichen und der vollstationären Pflege für große Teile der Betroffenen zu weitreichenden Veränderungen in der Lebens- und Versorgungssituation gekommen ist. Wenngleich die Versorgungssituation ein heterogenes Bild darstellt - sowohl zwischen den Versorgungsarten (ambulant und stationär) als auch innerhalb der Gruppe der pflegebedürftigen Personen selbst -, wurden Verschlechterungen in der pflegerischen und ärztlichen Versorgung wahrgenommen.

Für den ambulanten Versorgungsbereich zeigt sich, dass für einen Großteil der Pflegehaushalte essentielle Unterstuitzungsleistungen, professionell und informell, nur noch eingeschränkt zur Verfügung standen. Nach Angaben der Befragten hat sich besonders die psychische Gesundheit wie auch die geistige Fitness und Lebensqualität der pflegebedürftigen Menschen verschlechtert. Gleichzeitig fühlte sich jeder fünfte Pflegehaushalt in der Pandemie nicht gut unterstützt oder gibt an, die Pflege nicht gut bewältigen zu können.

Für das vollstationäre Setting werden die während der ersten Pandemie-Welle ganz erheblichen Einschränkungen der sozialen Teilhabe der pflegebedürftigen Menschen deutlich. Sowohl hinsichtlich der Möglichkeit, das eigene Zimmer oder die Pflegeeinrichtung zu verlassen, als auch mit Blick auf Kontakt zu den Bezugspersonen, ob persönlich oder telefonisch. Die eingeschränkten Möglichkeiten zur sozialen Teilhabe, Kontaktreduktion, soziale Isolation und reduzierte gesundheitliche Versorgung schlagen sich sodann auch in einer ausgeprägten Verschlechterung des von den Bezugspersonen wahrgenommenen Zustands der pflegebedürftigen Menschen nieder. 
Die verschlechterte Versorgungssituation und die Kontaktreduktion in den Pflegeeinrichtungen belastet zudem die befragten Bezugspersonen selbst - im Vergleich zu den ambulant Pflegenden sogar deutlich stärker. Ein Erklärungsansatz ist, dass dies auf die besonderen emotionalen Belastungen verweist, die daraus resultieren, dass in der vollstationären Pflege ein Abwägen von Schutzmaßnahmen und Selbstbestimmung nicht selbst vorgenommen werden konnte, sondern per gesetzlicher Anordnung - und zwar in der ersten Welle eindeutig zu Gunsten von Schutzpflichten erfolgte. Einhergehend mit verbesserten Hygienekonzepten, den Schnelltestmöglichkeiten ab Herbst 2020 sowie der Impfstrategie ab Januar 2021 bestehen seit der zweiten Welle mehr Möglichkeiten, Schutzbedarf sowie Kontakt- und Teilhabemöglichkeiten in anderer Form abzuwägen. Inwiefern dies gelungen ist, obliegt der Untersuchung im Rahmen weiterer Erhebungen.

In der Gesamtschau bestätigt die Untersuchung für den Kontext der ersten Welle, dass die Maßnahmen, die die pflegebedürftigen Menschen vor einer Ansteckung an COVID-19 schützen sollten, im Umkehrschluss zu Einschnitten in der pflegerischen und gesundheitlichen Versorgung der Pflegebedürftigen sowie zu stärkerer sozialer Isolation und Zunahme der psychischen Belastungen geführt haben. Alle Ergebnisse weisen darauf hin, dass derartige Entscheidungen, wie sie in der beginnenden Pandemie getroffen wurden, vor dem Hintergrund des heute vorliegenden Wissens zu reflektieren sind. Die Erkenntnisse gilt es für zukünftige Pandemiekonzepte zu berücksichtigen.

\section{Literatur}

Bundesregierung (2020) Videokonferenz der Bundeskanzlerin mit den Regierungschefinnen und Regierungschefs der Länder am 28. Oktober 2020. BESCHLUSS TOP Bekämpfung der SARS-Cov2-Pandemie. https:// www.bundesregierung.de/resource/blob/997532/ 1805024/5353edede6c0125ebe5b5166504dfd79/ 2020-10-28-mpk-beschluss-corona-data.pdf. Zugegriffen: 23. März 2021

Eggert S, Teuber C, Budnick A, Gellert P, Kuhlmey A (2020) Pflegende Angehörige in der COVID-19-Krise Ergebnisse einer bundesweiten Befragung. Zentrum für Qualität in der Pflege (ZQP)

Eggert S, Teubner C (2021) ZQP-Analyse. Die SARS CoV-2-Pandemie in der professionellen Pflege: Perspektive stationärer Langzeitpflege und ambulanter Dienste. Zentrum für Qualität in der Pflege (ZQP)

Gardner W, States D, Bagley N (2020) The Coronavirus and the risks to the elderly in long-term care. J Aging Soc Policy 32:310-315. https://doi.org/10. 1080/08959420.2020.1750543

Horn V, Schweppe C (2020) Häusliche Altenpflege in Zeiten von Corona. https://www.sozialpaedagogik. fb02.uni-mainz.de/files/2020/07/Studie_JGU_H\%C3 $\%$ A4usliche-Pflege-unter-Corona.pdf. Zugegriffen: 19. Aug. 2020

Infratest dimap (2020a) ARD-DeutschlandTREND Oktober 2020. Eine Studie im Auftrag der tagesthemen. https://www.tagesschau.de/inland/deutschlandtrend2347.pdf. Zugegriffen: 20. März 2021

Infratest dimap (2020b) ARD-DeutschlandTREND November 2020. Eine Studie zur politischen Stimmung im Auftrag der ARD-Tagesthemen und der Tageszeitung DIE WELT. https://www.infratest-dimap.de/ fileadmin/user_upload/DT2011_Bericht.pdf. Zugegriffen: 20. März 2021

Kantar (2019) Wissenschaftliche Evaluation der Umstellung des Verfahrens zur Feststellung der Pflegebedürftigkeit (§ 18c Abs. 2 SGB XI). Abschlussbericht. Los 2: Allgemeine Befragungen. https://www. bundesgesundheitsministerium.de/fileadmin/Dateien/ 3_Downloads/P/Pflegebeduerftigkeitsbegriff_

Evaluierung/Abschlussbericht_Los_2_Evaluation_ 18c_SGB_XI.pdf. Zugegriffen: 2. Apr. 2020 (Abschlussbericht für das Bundesministerium für Gesundheit (BMG) von Kantar Public Division Deutschland)

Kessler E-M, Strumpen S, Kricheldorff C, Franke A, Pantel J, Gellert P, Püllen R (2020a) Partizipation und soziale Teilhabe älterer Menschen trotz Corona-Pandemie ermöglichen. https://www.dgggonline.de/fileadmin/aktuelles/covid-19/20200424_ DGGG_Statement_Sektionen_II_III_IV_Soziale_ Teilhabe_und_Partizipation.pdf. Zugegriffen: 19. Aug. 2020 (Gemeinsames Statement der Sektionen für Geriatrische Medizin (II), Sozialund Verhaltenswissenschaftliche Gerontologie (III), Soziale Gerontologie und Altenhilfe (IV) der Deutschen Gesellschaft für Gerontologie und Geriatrie (DGGG e. V.))

Kessler EM, Strumpen S, Kircheldorff C, Franke A, Pantel J, Gellert P (2020b) Partizipation und soziale Teilhabe älterer Menschen trotz Corona-Pandemie ermöglichen (Gemeinsames Statement der Sektionen für 
Geriatrische Medizin (II), Sozial- und Verhaltenswissenschaftliche Gerontologie (III), Soziale Gerontologie und Altenhilfe (IV) der Deutschen Gesellschaft für Gerontologie und Geriatrie (DGGG e. V.).)

Müller O, Neuhann F, Razum O (2020) Epidemiologie und Kontrollmaßnahmen bei COVID-19. Dmw - Dtsch Medizinische Wochenschrift 145:670-674. https://doi.org/10.1055/a-1162-1987

Räker M, Schwinger A, Klauber J (2020) Was leisten ambulante Pflegehaushalte? Eine Befragung zu Eigenleistungen und finanziellen Aufwänden. In: Jacobs K, Kuhlmey A, Greß S, Klauber J, Schwinger A (Hrsg) Pflege-Report 2020: Neuausrichtung von Versorgung und Finanzierung. Springer Berlin Heidelberg, Berlin, Heidelberg, S 65-95

Röhr S, Reininghaus U, Riedel-Heller S (2020) Mental and social health in the German old age population largely unaltered during COVID-19 lockdown: results of a representative survey

Rothgang H et al (2020) Pflege in Zeiten von Corona: Zentrale Ergebnisse einer deutschlandweiten Querschnittsbefragung vollstationarer Pflegeheime. Pflege 33:265-275. https://doi.org/10.1024/1012-5302/ a000760
Schmidt B (2018) Melderegister, Mixed-Mode und Teilnahmeanreize: ein Modell zur Rekrutierung von Teilnehmern für sozialwissenschaftliche Panelbefragungen. Dissertation zur Erlangung der Doktorwürde durch den Promotionsausschuss Dr. rer. pol. der Universität Bremen (https://media.suub.uni-bremen. de/bitstream/elib/1403/1/00106477-1.pdf. Zugegriffen: 20. April 2021)

Statistisches Bundesamt (2018) Pflegestatistik. Pflege im Rahmen der Pflegeversicherung. Ländervergleich - Pflegebedürftige. https://www.destatis. de/DE/Themen/Gesellschaft-Umwelt/Gesundheit/ Pflege/Publikationen/Downloads-Pflege/laenderpflegebeduerftige-5224002179004.pdf?_blob= publicationFile\&v=5. Zugegriffen: 6. Jan. 2020

Statistisches Bundesamt (2019) Bildung. Statistisches Jahrbuch 2019. DeStatis, Wiesbaden, S 85-126

Statistisches Bundesamt (2020) Einkommen, Einnahmen und Ausgaben privater Haushalte 2019 in den Gebietsständen. Ergebnisse der Laufenden Wirtschaftsrechnungen (LWR). https://www.destatis.de/DE/ Themen/Gesellschaft-Umwelt/Einkommen-KonsumLebensbedingungen/Einkommen-EinnahmenAusgaben/Tabellen/gebietsstaende-lwr.html. Zugegriffen: 6. März 2020

Open Access Dieses Kapitel wird unter der Creative Commons Namensnennung 4.0 International Lizenz (http:// creativecommons.org/licenses/by/4.0/deed.de) veröffentlicht, welche die Nutzung, Vervielfältigung, Bearbeitung, Verbreitung und Wiedergabe in jeglichem Medium und Format erlaubt, sofern Sie den/die ursprünglichen Autor(en) und die Quelle ordnungsgemäß nennen, einen Link zur Creative Commons Lizenz beifügen und angeben, ob Änderungen vorgenommen wurden.

Die in diesem Kapitel enthaltenen Bilder und sonstiges Drittmaterial unterliegen ebenfalls der genannten Creative Commons Lizenz, sofern sich aus der Abbildungslegende nichts anderes ergibt. Sofern das betreffende Material nicht unter der genannten Creative Commons Lizenz steht und die betreffende Handlung nicht nach gesetzlichen Vorschriften erlaubt ist, ist für die oben aufgeführten Weiterverwendungen des Materials die Einwilligung des jeweiligen Rechteinhabers einzuholen.

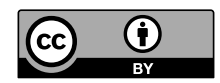

